\title{
A data-driven analysis on bridging techniques for heterogeneous materials and structures
}

\author{
Qian Shao, Jian Liu, Qun Huang, Jie Yang, Heng Hu, Salim Belouettar \& \\ Gaetano Giunta
}

To cite this article: Qian Shao, Jian Liu, Qun Huang, Jie Yang, Heng Hu, Salim Belouettar \& Gaetano Giunta (2019): A data-driven analysis on bridging techniques for heterogeneous materials and structures, Mechanics of Advanced Materials and Structures, DOI:

10.1080/15376494.2018.1546415

To link to this article: https://doi.org/10.1080/15376494.2018.1546415

\section{Published online: 18 Jan 2019.}

Submit your article to this journal $\llbracket$

山 Article views: 209

Q View related articles $\sqsubset$

View Crossmark data ־

4 Citing articles: 1 View citing articles $\pi$ 


\title{
A data-driven analysis on bridging techniques for heterogeneous materials and structures
}

\author{
Qian Shao ${ }^{a}$, Jian Liu ${ }^{a}$ (D), Qun Huang ${ }^{a}$, Jie Yang ${ }^{a}$, Heng Hua , Salim Belouettar ${ }^{b}$, and Gaetano Giunta ${ }^{b}$ \\ ${ }^{a}$ School of Civil Engineering, Wuhan University, Wuhan, PR China; ${ }^{b}$ Luxembourg Institute of Science and Technology, \\ Esch-sur-Alzette, Luxembourg
}

\begin{abstract}
A bridging technique based on Lagrange multipliers, namely the Arlequin method, is widely used for coupling multi-scale models. However, the definition of the following key parameters is still unclear: the energy partition functions, the characteristic length of the coupling operator and the size of the coupling zone. This work aims to investigate the influences of these factors on the coupling accuracy by conducting global sensitivity analysis on different multi-scale models. To this end, a data-driven model that approximates the input/output behavior of the numerical model is built by using the Sparse Polynomial Chaos Expansion (SPCE) methodology. Then, Sobol' indices that quantify the sensitivity of the input factors are calculated analytically from the data-driven model with a negligible additional computational cost. Interaction effects among different parameters are also captured. Using this approach, several benchmark tests, including a coarse-fine bar model, a particle-continuum model and a 2D-1D sandwich model, are considered to explore the optimal settings of the coupling factors, which hopefully help for the multi-scale analysis of heterogeneous materials and structures.
\end{abstract}

\section{ARTICLE HISTORY}

Received 4 November 2018

Accepted 5 November 2018

\section{KEYWORDS}

Arlequin method; datadriven analysis; global sensitivity analysis; sparse polynomial chaos expansion; multi-scale models; heterogeneous materials and structures

\section{Introduction}

Multi-scale strategies have been widely used over the past few decades to improve the accuracy and efficiency of modeling and simulation of engineering problems. In multi-scale computations, a bridging technique is necessary for connecting heterogeneous models, such as the coupling between atomistic and continuum models, or between finite element models with incompatible meshes, etc. Many efforts have been devoted to the development of coupling techniques, for instance the handshake method [1], the bridging scale decomposition [2], the bridging domain method [3], the Arlequin method [4] and so on.

Due to the flexibility and universality, the Arlequin method and its variants have been widely used [4-11]. The Arlequin method was firstly proposed by Ben Dhia [4] and it allows the superposition of various mechanical models. By means of energy partition functions and Lagrange multipliers, different models are glued in a coupling zone. Ben Dhia et al. [6] further assessed the effectiveness of the Arlequin method for coupling various finite element models in one, two and three dimensions. Hu et al. [12-15] successfully applied the Arlequin method to the multi-scale modeling of sandwich structures. He et al. [16] applied the Arlequin method to couple variable order beam elements. Whilst Biscani et al. [17] employed the Arlequin method for coupling variable kinematic plate elements. The coupling between solid and higher-order plate finite elements was established by Biscani et al. [18]. An Arlequin-based method was also used to couple continuum and molecular dynamics systems in [19]. Kpogan et al. [20, 21] applied the Arlequin method to simulate the buckling phenomena of a rolled thin sheet. Qiao et al. [22] successfully implemented the Arlequin method into the commercial finite element software ABAQUS to couple the same type elements with different grid density or various types of elements.

As mentioned in [6], the optimal practical settings of the weighting parameter functions, the size of the superposition zone and the multiple superposition procedure are essential for a multi-scale analysis by the Arlequin method. Since then, many efforts have been devoted to explore the choices of these Arlequin parameters. In [8], Ben Dhia offered some qualitative suggestions for the choice of the weight parameter functions and the coupling operators. Considering the applications of these methods to the coupling of the particle-continuum model, the influence of coupling operators $L^{2}$ and $H^{1}$ were discussed in [9], whilst the convergence of the solution as well as the effect of the location and the size of the overlapping region were analyzed by Prudhomme et al. [10]. To avoid the drawback of mesh dependency, an improved version of the Arlequin method for blending particle and continuum models was proposed in [11]. Guidault and Belytschko [23] indicated that the $H^{1}$ coupling operator benefits from the derivatives of displacement, which allows to perfectly blend a very coarse mesh with a very fine one. 
$\Omega$

\begin{tabular}{lllll}
\multicolumn{3}{c}{$\boldsymbol{\Omega}$} & \\
\hline & $\boldsymbol{\Omega}_{f}$ & & & \\
& $\boldsymbol{\Omega}_{s}$ & $\boldsymbol{\Omega}_{c}$ \\
& &
\end{tabular}

Figure 1. The Arlequin method in a general mechanical problem.

Sun and Mota [24] found that the $H^{1}$ coupling is more reliable to enforce weak compatibility than $L^{2}$ when the Lagrange multiplier and the coarse deformation mapping share the same discretization. Hu et al. [25] proposed a new coupling operator, namely $H_{p}^{1}$ that permits to obtain a better conditioning of the global stiffness matrix than $H^{1}$ or $L^{2}$ coupling operators when modeling sandwich structures.

Although the Arlequin method has been widely used, a quantitative guidance for setting the above mentioned parameters remains unclear. Global Sensitivity Analysis (GSA) is a reliable tool that helps quantitatively explore the respective effect of each input parameter onto the response of a physical model and further seek for regions in the space of input factors where the model output meets the optimum criterion [26]. In this work, GSA is performed to explore the quantitative influence of the parameters on multi-scale modeling accuracy. A wide range of metrics and techniques have been developed to perform GSA, such as linear regression-based methods, variance-based methods [27], etc. Among others, Sobol' indices are widely used to measure the impact of model parameters [28]. Typically, two approaches can be used to evaluate Sobol' indices, involving Monte-Carlo methods and metamodeling methods. The latter have received much attention due to their effectiveness and high-efficiency [29-31]. Following metamodeling approach, we build up a data-driven model that approximates the input/output behavior of the original numerical model. Then, Sobol' indices can be calculated analytically from the data-driven model with a negligible additional computational cost. To "train" the data-driven model, the Sparse Polynomial Chaos Expansion methodology, which uses orthogonal polynomials to approximate the response surface, has been commonly used. The idea of SPCE was first proposed by Blatman and Sudret [29-32], and further developed by $\mathrm{Hu}$ and Youn [33], Fajraoui et al. [34], Shao et al. [35], etc. In this work, we use the algorithm proposed by Shao et al. [35] to approximate the data-driven model with SPCE. This algorithm is based on Bayesian model averaging. The proposed algorithm uses the Kashyap information criterion (KIC) [36] to select the best SPCE model for a given input/output sample. Here, the weight functions, the characteristic length and the length of the coupling zone involving in the Arlequin method are considered as inputs for multi-scale models, whilst the relative errors between the numerical solution and the reference solution are considered as outputs. This strategy can be referred as a data-driven approach since the settings of Arlequin parameters are explored by using a large number of numerical experiments instead of intuition or personal experience.

The layout of this article is as follows. In Section 2, the Arlequin method is briefly recalled. Section 3 reviews the Sobol' decomposition and Bayesian SPCE, followed by the introduction of the framework for data-driven analysis on the Arlequin method. In Section 4, three classical multi-scale models coupled by the Arlequin method are analyzed by the proposed procedure. Finally, conclusions on the selection of the coupling factors are drawn in Section 5.

\section{The Arlequin method}

In this section, the basic idea of the Arlequin method $[4,5]$ is recalled. As shown in Figure 1, the considered domain $\Omega$ of a mechanical system is split into two sub-domains with an overlapping zone: domain $\Omega_{f}$ analyzed by a fine model and domain $\Omega_{c}$ by a coarse model. The resulting intersecting zone is represented by $\Omega_{s}$, where $\Omega_{s}=\Omega_{f} \cap \Omega_{c}$.

According to the Arlequin framework, the energy of the domain $\Omega$ is obtained by adding the energy of $\Omega_{f}$ and $\Omega_{c}$. The energy $E_{k}$ for each sub-domain reads:

$$
E_{k}\left(\boldsymbol{u}_{k}\right)=\frac{1}{2} \int_{\Omega_{k}} \alpha_{k} \boldsymbol{\sigma}\left(\boldsymbol{u}_{k}\right): \varepsilon\left(\boldsymbol{u}_{k}\right) d \Omega_{k}-\int_{\Omega_{k}} \beta_{k} \boldsymbol{f}_{k} \cdot \boldsymbol{u}_{k} d \Omega_{k}
$$

where $\boldsymbol{u}_{k}$ and $\boldsymbol{f}_{k}$ represent the displacement and the external force per unit volume in $\Omega_{k}$ with $k=f$ or $c$. To avoid considering twice the energy of the total system in the coupling zone, the weight functions $\alpha_{k}$ and $\beta_{k}$ in Eq. (1) satisfy the following relations:

$$
\begin{cases}\alpha_{f}=\beta_{f}=1, & \text { in } \Omega_{f} \backslash \Omega_{s}, \\ \alpha_{c}=\beta_{c}=1, & \text { in } \Omega_{c} \backslash \Omega_{s}, \\ \alpha_{f}+\alpha_{c}=\beta_{f}+\beta_{c}=1, & \text { in } \Omega_{s} .\end{cases}
$$

They are usually assumed to be positive piecewise continuous functions, for example, constant, linear, quadratic and so on. In this article, we limit these weight functions to be constant in the gluing zone as in Ben Dhia et al. [6] and $\mathrm{Hu}$ et al. [25].

To ensure the displacement continuity in the coupling zone $\Omega_{s}$, the Lagrange multiplier $\lambda$ is introduced as a fictive gluing force. Here, we consider an $H^{1}$-type coupling operator as analyzed in [4] that possesses good mathematical and numerical properties:

$$
\mathcal{C}\left(\boldsymbol{\lambda}, \boldsymbol{u}_{k}\right)=\int_{\Omega_{s}}\left(\boldsymbol{\lambda} \cdot \boldsymbol{u}_{k}+\ell^{2} \varepsilon(\boldsymbol{\lambda}): \varepsilon\left(\boldsymbol{u}_{k}\right)\right) d \boldsymbol{\Omega}
$$

The characteristic length $\ell$ in Eq. (3) is a positive parameter homogeneous to a length, $\varepsilon(\boldsymbol{\lambda})$ and $\varepsilon\left(\boldsymbol{u}_{\boldsymbol{k}}\right)$ are the gradients terms. One should note that the coupling operator in Eq. (3) tends to be a $L^{2}$-type one when the characteristic length $\ell=0$, see [6].

For the whole problem, searching the variables $\boldsymbol{u}_{f}$ and $\boldsymbol{u}_{c}$ is equivalent to minimizing the following functional:

$$
\mathscr{T}\left(\boldsymbol{u}_{f}, \boldsymbol{u}_{c}, \boldsymbol{\lambda}\right)=E_{f}\left(\boldsymbol{u}_{f}\right)+E_{c}\left(\boldsymbol{u}_{c}\right)+\mathcal{C}\left(\boldsymbol{\lambda}, \boldsymbol{u}_{f}-\boldsymbol{u}_{c}\right)
$$

We derive the discrete formulations from the continuous one Eq. (4) by using the finite element method. The minimization of Eq. (4) leads to the following linear system:

$$
\left[\begin{array}{ccc}
\boldsymbol{K}_{f} & 0 & -\boldsymbol{C}_{f} \\
0 & \boldsymbol{K}_{c} & \boldsymbol{C}_{c} \\
-\boldsymbol{C}_{f}^{T} & \boldsymbol{C}_{c}^{T} & 0
\end{array}\right]\left[\begin{array}{c}
\boldsymbol{U}_{f} \\
\boldsymbol{U}_{c} \\
\Lambda
\end{array}\right]=\left[\begin{array}{c}
\boldsymbol{F}_{f} \\
\boldsymbol{F}_{c} \\
0
\end{array}\right]
$$

where $\boldsymbol{U}_{f}, \boldsymbol{U}_{c}$, and $\Lambda$ represent the global displacement vector in $\Omega_{f}, \Omega_{c}$, and the global Lagrange multiplier field, 


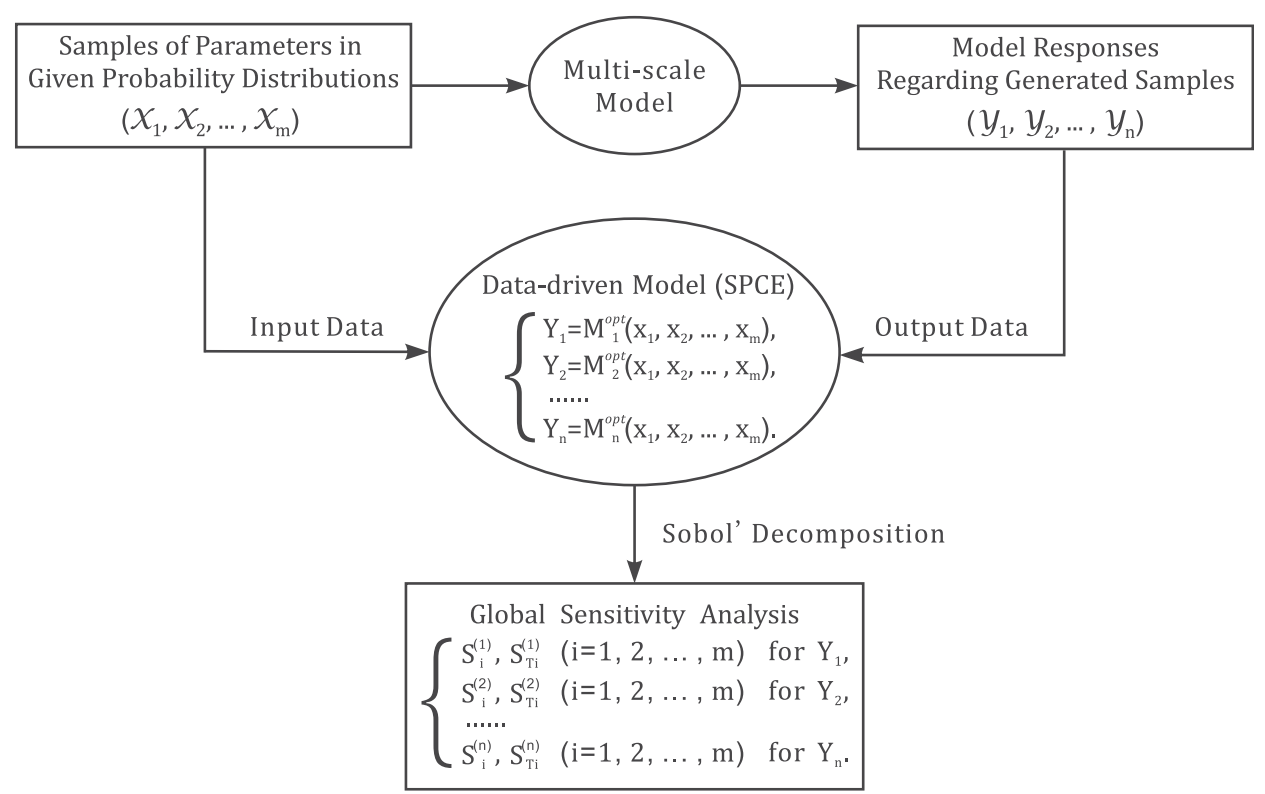

Figure 2. The process of data-driven analysis for the Arlequin method.

respectively. Solving the linear system Eq. (5) gives the displacement field of each sub-domain. The displacement field $\boldsymbol{U}$ of the multi-scale model can be then expressed as:

$$
\boldsymbol{U}=\alpha_{f} \boldsymbol{U}_{f}+\alpha_{c} \boldsymbol{U}_{c}
$$

\section{Global sensitivity analysis}

In this work, GSA is performed by using a variance-based method. By Sobol' decomposition, the Sobol' indices are derived to evaluate the sensitivity of model responses to the input variables. To calculate Sobol' indices efficiently, SPCE is adopted to represent the input/output relations of the original model. The construction of SPCE is based on the theory of Bayesian model averaging. In the following, we recall briefly the Sobol' decomposition and the Baysian SPCE. Then the procedure of datadriven analysis on the Arlequin method is introduced.

\subsection{Sobol' decomposition}

Let us consider a mathematical model having input vector $\boldsymbol{x}=\left(x_{1}, x_{2}, \ldots, x_{n}\right)^{T}$ and a scalar output $Y$ :

$$
Y=M(\boldsymbol{x}), \quad \boldsymbol{x} \in \mathbb{K}^{n}
$$

where the input parameters are independent random variables uniformly distributed in $[0,1]$ :

$$
\boldsymbol{x}=\left\{x_{1}, \ldots, x_{n}\right\}, \quad x_{i} \sim \mathcal{U}[0,1], \quad i=1, \ldots, n
$$

We assume that $Y$ is square integrable, that is $Y \in \mathcal{L}^{2}$. The unique Sobol' decomposition of $M(\boldsymbol{x})$ into summands of increasing dimension reads [28]:

$$
M(\boldsymbol{x})=M_{0}+\sum_{i_{1}=1}^{n} M_{i_{1}}\left(x_{i_{1}}\right)+\sum_{i_{2}>i_{1}}^{n} M_{i_{1} i_{2}}\left(x_{i_{1}}, x_{i_{2}}\right)+\cdots+M_{12 \ldots n}(\boldsymbol{x})
$$

where the integral of each summand $M_{i_{1} \ldots i_{s}}\left(x_{i_{1}}, \ldots, x_{i_{s}}\right)$ over any of its independent variables is zero, that is:

$$
\int_{0}^{1} M_{i_{1} \ldots i_{s}}\left(x_{i_{1}}, \ldots, x_{i_{s}}\right) d x_{i_{k}}=0, \quad 1 \leqslant k \leqslant s
$$

Due to Eq. (10), the summands of the decomposition are orthogonal to each other in the following sense:

$\int_{\mathbb{K}^{n}} M_{i_{1} \ldots i_{s}}\left(x_{i_{1}}, \ldots, x_{i_{s}}\right) M_{j_{1} \ldots j_{t}}\left(x_{j_{1}}, \ldots, x_{j_{t}}\right) d \boldsymbol{x}=0 \quad$ for $\quad\left\{i_{1} \ldots i_{s}\right\} \neq\left\{j_{1} \ldots j_{t}\right\}$

where $d x=d x_{1} \ldots d x_{n}$ for the sake of simplicity.

Due to the orthogonality property in Eq. (11), the total variance of $M(\boldsymbol{x})$ can be decomposed as follows:

$$
D=\int_{\mathbb{K}^{n}} M^{2}(\boldsymbol{x}) d \boldsymbol{x}-M_{0}^{2}=\sum_{i_{1}=1}^{n} D_{i_{1}}+\sum_{i_{2}>i_{1}}^{n} D_{i_{1} i_{2}}+\cdots+D_{12 \ldots n}
$$

where the partial variance $D_{i_{1} \ldots i_{s}}$ reads:

$$
D_{i_{1} i_{2} \ldots i_{s}}=\int_{\mathbb{K}^{s}} M_{i_{1} \ldots i_{s}}^{2}\left(x_{i_{1}}, \ldots, x_{i_{s}}\right) d x_{i_{1}} \ldots d x_{i_{s}}
$$

The Sobol' indices are defined as follows:

$$
S_{i_{1} \ldots i_{s}}=\frac{D_{i_{1} \ldots i_{s}}}{D} \in[0,1]
$$

Obviously, they satisfy:

$$
\sum_{i_{1}=1}^{n} S_{i_{1}}+\sum_{i_{2}>i_{1}}^{n} S_{i_{1} i_{2}}+\cdots+S_{12 \ldots n}=1
$$

The first-order sensitivity index $S_{i}$ represents the influence due to $x_{i}$ alone. $S_{i j}$ measures the amount of variance of $Y$ due to the cooperative effect of $x_{i}$ and $x_{j}$. Similarly, $S_{i_{1} i_{2} \ldots i_{s}}$ describes the sensitivity due to the set of input parameters $\left\{x_{i_{1}}, x_{i_{2}}, \ldots, x_{i_{s}}\right\}$. The closer the Sobol' index to 1 , the more sensitive the corresponding parameters. In this work, the first-order sensitivity index $S_{i}$ and the total sensitivity index $S_{T i}$ are used to evaluate the sensitivity of the coupling parameters. $S_{T i}$ evaluates the total contribution of $x_{i}$ to the 


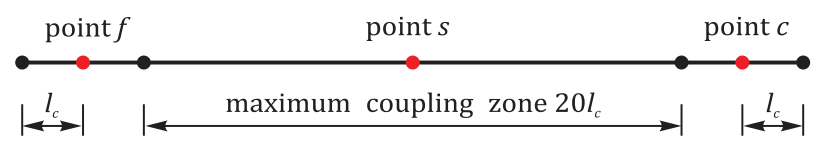

Figure 3. The location of the selected points for error evaluation.

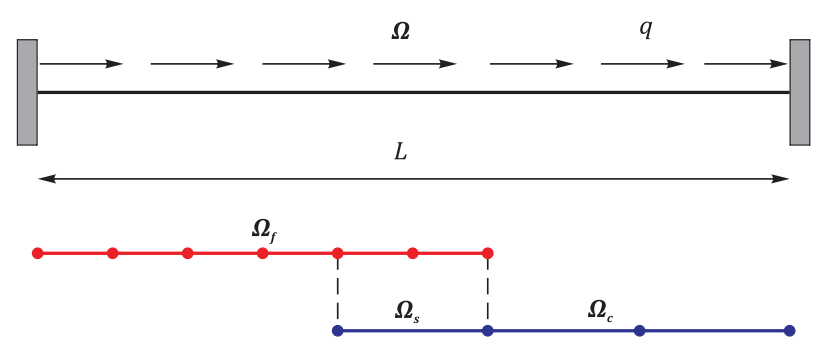

Figure 4. Sketch for a bar clamped at both ends.

Table 1. Parameters of the coarse-fine bar model.

\begin{tabular}{lccc}
\hline$E(\mathrm{~Pa})$ & $L(\mathrm{~m})$ & $I_{c}\left(10^{-1} \mathrm{~m}\right)$ & $I_{f}\left(10^{-2} \mathrm{~m}\right)$ \\
\hline 100 & 3 & 1.25 & 2.5 \\
\hline
\end{tabular}

Table 2. The range of parameters considered for GSA.

\begin{tabular}{ccccc}
\hline & $\alpha_{c}$ & $\beta_{c}$ & $\ell$ & $L_{s}$ \\
\hline case 1 & {$[0,1]$} & {$[0,1]$} & {$[0, L]$} & {$\left[2 I_{c}, 20 I_{c}\right]$} \\
case 2 & {$[0,0.5]$} & {$[0,0.5]$} & {$[0, L]$} & {$\left[2 I_{c}, 20 I_{c}\right]$} \\
\hline
\end{tabular}

variance of $Y$ in the following form:

$$
S_{T i}=\sum_{r: i \in \boldsymbol{r}} S_{\boldsymbol{r}}=1-S_{\sim i}
$$

where the symbol “ $\sim$ " means "complementary of" in the sequel.

In order to calculate the Sobol' indices in a feasible and effective way, the SPCE of the model response is introduced in the next subsection.

\subsection{Bayesian sparse polynomial chaos expansion of the model response}

The model response can be expanded in terms of a polynomial basis as follows:

$Y=M(\boldsymbol{x}) \equiv \sum_{\boldsymbol{b} \in \mathbb{N}^{n}} a_{\boldsymbol{b}} \psi_{\boldsymbol{b}}(\boldsymbol{x})$, with $\psi_{\boldsymbol{b}}(\boldsymbol{x})=\psi_{b_{1} \ldots b_{n}}(\boldsymbol{x})=\prod_{i=1}^{n} \psi_{b_{i}}\left(x_{i}\right)$

where $\boldsymbol{b}=b_{1} \ldots b_{n} \quad\left(b_{i} \in \mathbb{N}, 1 \leqslant i \leqslant n\right) \quad$ is an $n$-dimensional index, and the $a_{b}$ 's are the polynomial coefficients.

Equation (17) is usually referred to as the polynomial chaos expansion (PCE) of $Y$. A polynomial basis function is a cumulative product of $n$ univariate standardized shiftedLegendre polynomials, where $b_{i}$ the $i$-th element of $\boldsymbol{b}$ denotes the order of the Legendre polynomial in $x_{i}$. For instance, the first three Legendre polynomials are: $\psi_{0}(x)=$ $1, \psi_{1}(x)=\sqrt{3}(2 x-1), \psi_{2}(x)=\frac{3 \sqrt{5}}{2}(2 x-1)^{2}-\frac{\sqrt{5}}{2}$.

For computational purposes, the PCE is usually truncated to retain a finite number of terms. A truncated PCE whose total degree $|\boldsymbol{b}| \equiv \sum_{i=1}^{n} b_{i}$ does not exceed a given order $p$ reads:

$$
Y \simeq M_{p}(\boldsymbol{x}) \equiv \sum_{\boldsymbol{b} \in A^{p, n}} a_{\boldsymbol{b}} \psi_{\boldsymbol{b}}(\boldsymbol{x}), A^{p, n} \equiv\left\{\boldsymbol{b} \in \mathbb{N}^{n}:|\boldsymbol{b}| \leqslant p\right\}
$$

To reduce the number of model evaluations and to prevent overfitting, a SPCE is usually adopted. A SPCE retains a small number of terms to capture the main feature of the model response:

$$
Y \simeq M_{A}(\boldsymbol{x}) \equiv \sum_{\boldsymbol{b} \in A} a_{\boldsymbol{b}} \psi_{\boldsymbol{b}}(\boldsymbol{x}), A \subseteq A^{p, n} \text { and } \frac{\operatorname{card}(A)}{\operatorname{card}\left(A^{p, n}\right)} \ll 1
$$

where operator card provides the cardinality of a set.

In this work, the construction of SPCE is based on Bayesian model averaging. Suppose there are $N_{m}$ plausible competing SPCE models $M_{A_{k}}$ :

$$
M_{A_{k}} \equiv \sum_{\boldsymbol{b} \in A_{k}} a_{\boldsymbol{b}} \psi_{\boldsymbol{b}}(\boldsymbol{x})=\boldsymbol{a}_{k} \boldsymbol{\psi}_{k}, \quad k=1, \ldots, N_{m}
$$

where $\boldsymbol{a}_{k}$ and $\boldsymbol{\psi}_{k}$ denote the vector of polynomial coefficients and polynomial terms.

Let $\mathcal{X}, \mathcal{Y}$ represent the input and output data sets respectively. By estimating each model's posterior probability which represents the model's ability to reproduce the data set $(\mathcal{X}, \mathcal{Y})$, the best SPCE representation among the set $\left\{M_{A_{k}}, k=1, \ldots, N_{m}\right\}$ can be selected. The posterior probabilities $P\left(M_{A_{k}} \mid \mathcal{Y}\right)$ are given by Bayes' theorem:

$$
P\left(M_{A_{k}} \mid \mathcal{Y}\right)=\frac{P\left(\mathcal{Y} \mid M_{A_{k}}\right) P\left(M_{A_{k}}\right)}{\sum_{i=1}^{N_{m}} P\left(\mathcal{Y} \mid M_{A_{i}}\right) P\left(M_{A_{i}}\right)}
$$

where $P\left(M_{A_{k}}\right)$ is the prior probability. Usually, let $P\left(M_{A_{k}}\right)=$ $1 / N_{m}$ when the prior information is vague. The denominator in Eq. (21) is the normalization constant. $P\left(\mathcal{Y} \mid M_{A_{k}}\right)$ is the likelihood of the observed data, expressing the preference shown by the data for different models. Therefore, $P\left(\mathcal{Y} \mid M_{A_{k}}\right)$ is used as the Bayesian model evidence. By Taylor series expansion and Laplace approximation, we can finally deduce the Kashyap information criterion (KIC) [35]:

$$
\begin{aligned}
-2 \ln P\left(\mathcal{Y} \mid M_{A_{k}}\right) \simeq & -2 \ln P\left(\mathcal{Y} \mid M_{A_{k}}, \tilde{\boldsymbol{a}}_{k}\right)-2 \ln P\left(\tilde{\boldsymbol{a}}_{k} \mid M_{A_{k}}\right) \\
& -P_{k} \ln (2 \pi)+\ln \left(\left|C_{\tilde{\boldsymbol{a}} \tilde{\boldsymbol{a}}}\right|\right)=K I C_{k}
\end{aligned}
$$

where the mean of the posterior $\tilde{\boldsymbol{a}}_{k}$ is referred to as the maximum a posteriori estimate (MAP), which represents the most likely parameter set for model $M_{A_{k}}$. The covariance matrix $C_{\tilde{a} \tilde{a}}$ is estimated at the MAP solution (i.e. $\boldsymbol{a}_{k}=\tilde{\boldsymbol{a}}_{k}$ ).

By introducing some assumptions about the prior probability, the parameters $\tilde{\boldsymbol{a}}_{k}$ and $C_{\tilde{\boldsymbol{a}} \tilde{\boldsymbol{a}}}$ can be calculated in an iterative way. Then, $K I C_{k}$ of the competing model $M_{A_{k}}$ can also be calculated. Apparently, the best SPCE is the one with the smallest KIC. In the following, we write the best SPCE as $M_{A^{\text {opt }}}$. The details of the algorithm are presented in [35].

Now let us calculate Sobol' indices from the constructed SPCE $M_{A^{\text {opt }}}$. Write $M_{A^{\text {opt }}}$ in the form of the Sobol' decomposition with a symbol $I_{i_{1} \ldots i_{s}}$ defined as follows: 

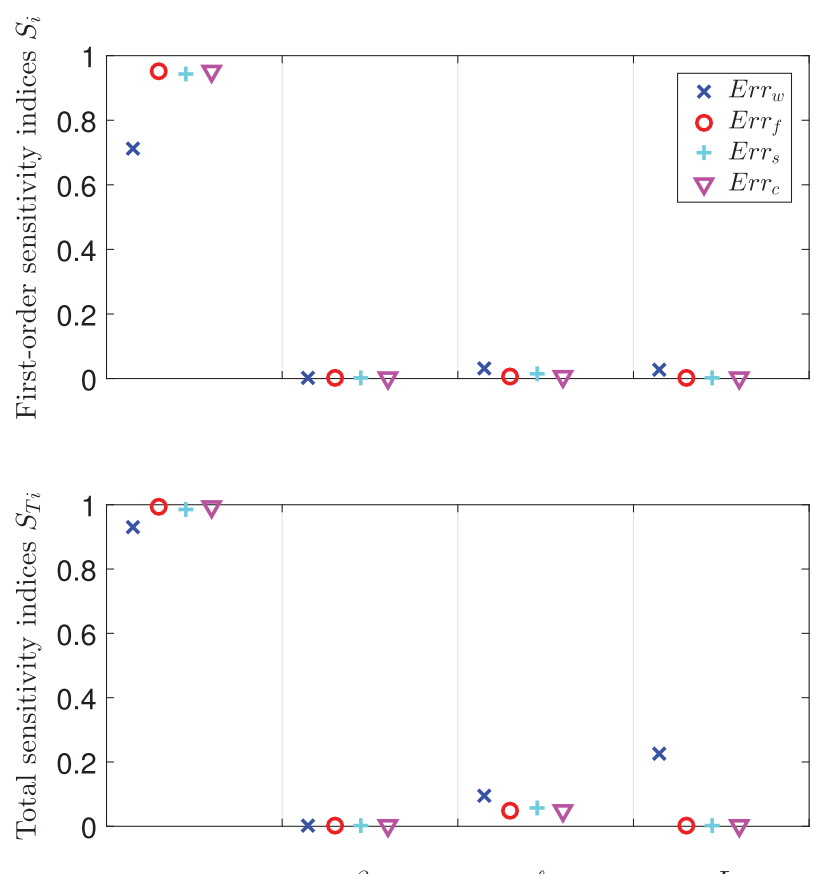

$\alpha_{c}$

$\beta_{c}$

(a)
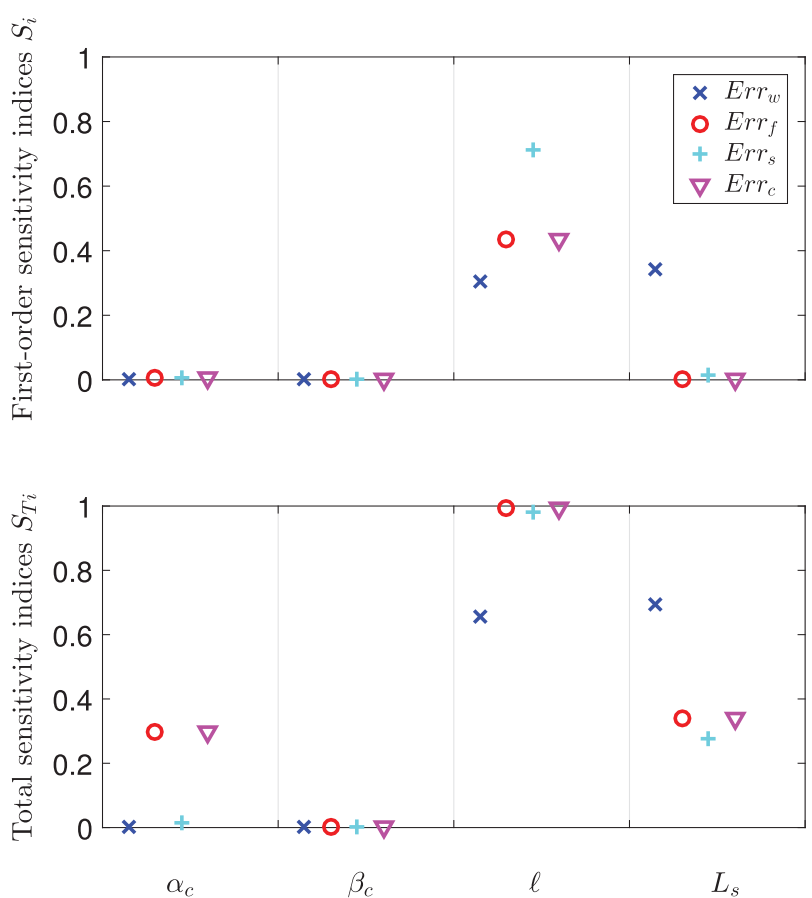

(b)

Figure 5. The Sobol' indices of (a) case 1 and (b) case 2 for the coarse-fine bar model.

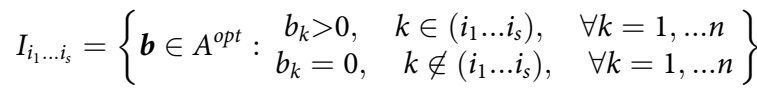

Then, the SPCE reads:

$$
\begin{aligned}
M_{A^{o p t}}= & a_{\boldsymbol{0}}+\sum_{i_{1}=1}^{n} \sum_{\boldsymbol{b} \in I_{i_{1}}} a_{\boldsymbol{b}} \psi_{\boldsymbol{b}}\left(x_{i_{1}}\right)+\sum_{i_{2}>i_{1}}^{n} \sum_{\boldsymbol{b} \in I_{i_{1} i_{2}}} a_{\boldsymbol{b}} \psi_{\boldsymbol{b}}\left(x_{i_{1}}, x_{i_{2}}\right) \\
& +\cdots+\sum_{i_{s}>\ldots>i_{1}}^{n} \sum_{\boldsymbol{b} \in I_{i_{1}, \ldots, s_{s}}} a_{\boldsymbol{b}} \psi_{\boldsymbol{b}}\left(x_{i_{1}}, \ldots, x_{i_{s}}\right) \\
& +\cdots+\sum_{\boldsymbol{b} \in I_{1, \ldots, n}} a_{\boldsymbol{b}} \psi_{\boldsymbol{b}}(\boldsymbol{x})
\end{aligned}
$$

where each summand in Eq. (9) can be identified in the above equation as follows: $M_{i_{1} \ldots i_{s}}\left(x_{i_{1}}, \ldots, x_{i_{s}}\right)=$ $\sum_{\boldsymbol{b} \in I_{i_{1} \ldots i_{s}}} a_{\boldsymbol{b}} \psi_{\boldsymbol{b}}\left(x_{i_{1}}, \ldots, x_{i_{s}}\right)$.

As long as a SPCE of a model response is built, it is easy to calculate the Sobol' indices analytically by the SPCE's coefficients. Due to the orthonormal property of the polynomial basis, the first-order and total Sobol' indices of input variables $x_{i}$ can be derived analytically according to Eqs. (13), (14), and (16) as follows: 
Table 3. The Sobol' indices of case 1 and case 2 for the coarse-fine bar model.

\begin{tabular}{|c|c|c|c|c|c|c|c|c|c|}
\hline & & \multicolumn{2}{|c|}{$\mathrm{Err}_{w}$} & \multicolumn{2}{|c|}{$E r r_{f}$} & \multicolumn{2}{|c|}{$\mathrm{Err}_{\mathrm{s}}$} & \multicolumn{2}{|c|}{$E r r_{c}$} \\
\hline & & $S_{i}$ & $S_{T i}$ & $S_{i}$ & $S_{T i}$ & $S_{i}$ & $S_{T i}$ & $S_{i}$ & $S_{T i}$ \\
\hline \multirow[t]{4}{*}{ case 1} & $\alpha_{c}$ & 0.7126 & 0.9318 & 0.9508 & 0.9938 & 0.9428 & 0.9843 & 0.9508 & 0.9938 \\
\hline & $\beta_{c}$ & 0 & 0 & 0 & 0 & 0 & 0 & 0 & 0 \\
\hline & $\ell$ & 0.0304 & 0.0947 & 0.0062 & 0.0492 & 0.0157 & 0.0572 & 0.0062 & 0.0492 \\
\hline & $L_{s}$ & 0.0258 & 0.2270 & 0 & 0 & 0 & 0 & 0 & 0 \\
\hline \multirow[t]{4}{*}{ case 2} & $\alpha_{c}$ & 0.0027 & 0.0027 & 0.0080 & 0.2966 & 0.0044 & 0.0134 & 0.0080 & 0.2966 \\
\hline & $\beta_{c}$ & 0 & 0 & 0 & 0 & 0 & 0 & 0 & 0 \\
\hline & $\ell$ & 0.3053 & 0.6554 & 0.4361 & 0.9920 & 0.7114 & 0.9796 & 0.4361 & 0.9920 \\
\hline & $L_{s}$ & 0.3419 & 0.6920 & 0 & 0.3376 & 0.0160 & 0.2761 & 0 & 0.3376 \\
\hline
\end{tabular}

$$
\begin{gathered}
S_{i}^{A^{o p t}}=\frac{D_{i}^{A^{o p t}}}{D^{A^{o p t}}}=\frac{\sum_{\boldsymbol{b} \in I_{i}} a_{\boldsymbol{b}}^{2}}{\sum_{\boldsymbol{b} \in A^{o p t} \backslash\{0\}} a_{\boldsymbol{b}}^{2}} \\
S_{T i}^{A^{\text {opt }}}=\sum_{\boldsymbol{b}: b_{i}>0} S_{\boldsymbol{b}}^{A^{\text {opt }}}
\end{gathered}
$$

\subsection{The proposed approach of data-driven analysis}

Here we propose a data-driven approach based on the Bayesian SPCE to analyze the settings of the Arlequin parameters. Suppose that we have " $m$ " Arlequin parameters to analyze, and " $n$ " model responses of interest. The computing process is sketched in Figure 2 and outlined as follows:

Step 1: Generate samples for the analyzed parameters in given probability distributions with $N_{d}$ realizations, e.g. $\mathcal{X}=$ $\left\{\mathcal{X}_{1}, \mathcal{X}_{2}, \ldots, \mathcal{X}_{m}\right\}$ where $\mathcal{X}_{i}=\left\{\mathcal{X}_{i}^{(1)}, \mathcal{X}_{i}^{(2)}, \ldots, \mathcal{X}_{i}^{\left(N_{d}\right)}\right\}^{T}$ with $1 \leq i \leq m$. There are several sampling methods, such as Latin Hypercube sampling, quasi-random low discrepancy sequences and quasi-Monte Carlo (QMC) method, etc. In this work, the input data set is generated by QMC method for its excellent property in space filling.

Step 2: Evaluate the multi-scale model at each group of design points using numerical methods. The corresponding model responses of interest are gathered into the vectors, say $\mathcal{Y}_{j}=\left\{\mathcal{Y}_{j}^{(1)}, \mathcal{Y}_{j}^{(2)}, \ldots, \mathcal{Y}_{j}^{\left(N_{d}\right)}\right\}^{T}$ with $1 \leq j \leq n$. To assess the effectiveness of the coupling, the relative errors between the coupling solution and the reference solution are chosen as the model responses.

Step 3: Transform the data $\left(\mathcal{X}, \mathcal{Y}_{j}\right) \quad(1 \leq j \leq n)$ into standardized form $\left(\boldsymbol{x}, \boldsymbol{y}_{j}\right) \quad(1 \leq j \leq n)$. And construct the data-driven model for each model response $Y_{j}$ from the data set $\left(\boldsymbol{x}, \boldsymbol{y}_{j}\right)$. The SPCE based on the theory of Bayesian model averaging is used to approximate the input/output relations of the multi-scale model. Thus, the optimal SPCE for each model response is written as $Y_{j}=$ $M_{j}^{o p t}\left(x_{1}, x_{2}, \ldots, x_{m}\right)(1 \leq j \leq n)$.

Step 4: Perform GSA on the data-driven model instead of the multi-scale model. Sobol' decomposition is easily conducted on the SPCE model. And subsequently the Sobol' indices for each Arlequin parameter are computed analytically. For every model response $Y_{j}$, we calculate both the first-order sensitivity indices $S_{i}^{(j)}$ and the total sensitivity indices $S_{T_{i}}^{(j)}$ to account for interactions between parameters.

\section{Numerical examples}

In this section, three typical examples using the Arlequin method are analyzed by the proposed approach: a coarsefine bar model, a particle-continuum model and a 2D-1D sandwich model. The coarse-fine bar model is a widely studied benchmark for the Arlequin method, see Ben Dhia et al. [6, 8], Guidault and Belytschko [37], Sun and Alejandro [24], etc. This benchmark test is firstly considered to verify the effectiveness of the approach. Then a more complex example, the particle-continuum model is studied. This is also a popular benchmark for the Arlequin method which has been studied by Bauman and Ben Dhia et al. [9], Prudhomme et al. [10], Chamoin et al. [38] when considering heterogeneous materials. At last, an example accounting a heterogeneous structure [25] is investigated.

In all cases, the coupling zone is always located at the center of the whole domain, which implies that the subdomains of the multi-scale model possess the same size. Suppose $L$ is the size of the whole domain. For the sake of simplicity, we define the element length of the coarse model $l_{c}$ as:

$$
l_{c}=\frac{L}{n}, \quad n \in \mathbb{N}^{*}
$$

and the size of the coupling zone $L_{s}$ is defined as:

$$
L_{s}=2 n_{s} l_{c}, \quad n_{s} \in \mathbb{N}^{*} \text { and } 2 n_{s} \leq n-4
$$

In this work, we set $n=24$. Thus, the maximum range of $2 n_{s}$ is $[2,20]$.

For each model, the energy partition functions $\alpha_{c}$ and $\beta_{c}$, the characteristic length of the coupling operator $\mathcal{L}$ and the size of the coupling zone $L_{s}$ are collected in the input variables $\boldsymbol{X}=\left\{\alpha_{c}, \beta_{c}, \ell, L_{s}\right\}$, whilst the model responses $\boldsymbol{Y}=$ $\left\{E r r_{w}, E r r_{f}, E r r_{s}, E r r_{c}\right\}$ denote the relative errors of displacements at specific points and can be calculated as follows:

$$
E r r_{j}=\left|\frac{\boldsymbol{U}-\boldsymbol{U}_{r e f}}{\boldsymbol{U}_{r e f}}\right|_{j}, \quad j=w, f, s, c
$$

where $\boldsymbol{U}_{\text {ref }}$ denotes the reference solution of displacement. $E r r_{w}$ is the maximum relative error of the whole domain. $E r r_{f}, E r r_{s}, E r r_{c}$ are respectively the relative errors at $l_{c}$ near the left end, in the middle, and at $l_{c}$ near the right end of the domain (see Figure 3). Since the maximum range of the coupling zone is $\left[2 l_{c}, 20 l_{c}\right]$, it is obvious that with the change of coupling zone, point $f$ and point $c$ always locate at $\Omega_{f}$ and $\Omega_{c}$, respectively, while point $s$ is always in the coupling 

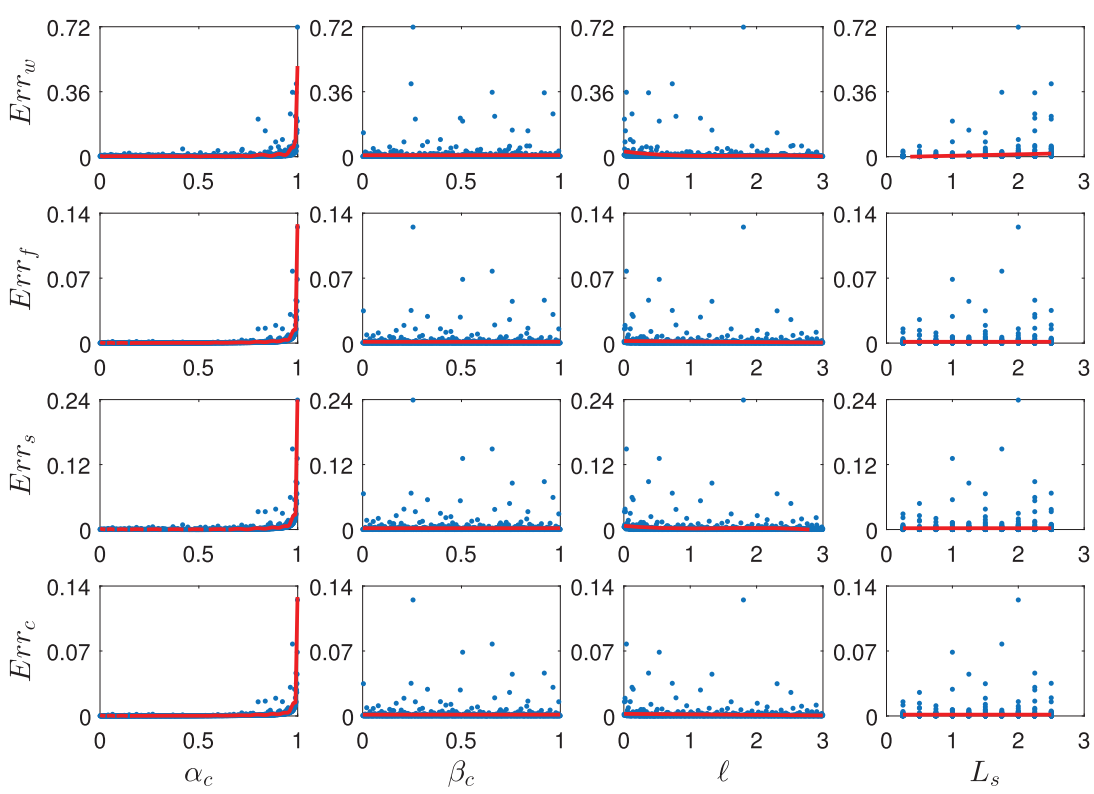

(a)
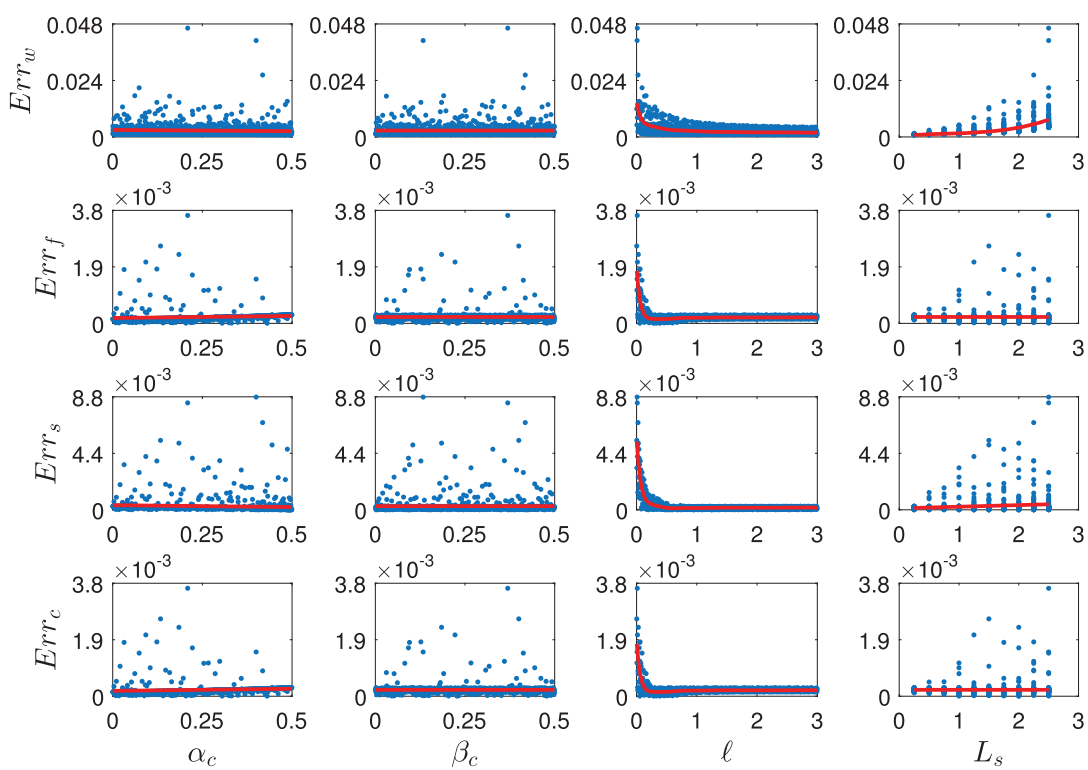

(b)

Figure 6. The marginal effect of Arlequin parameters in (a) case 1 and (b) case 2 for the coarse-fine bar model.
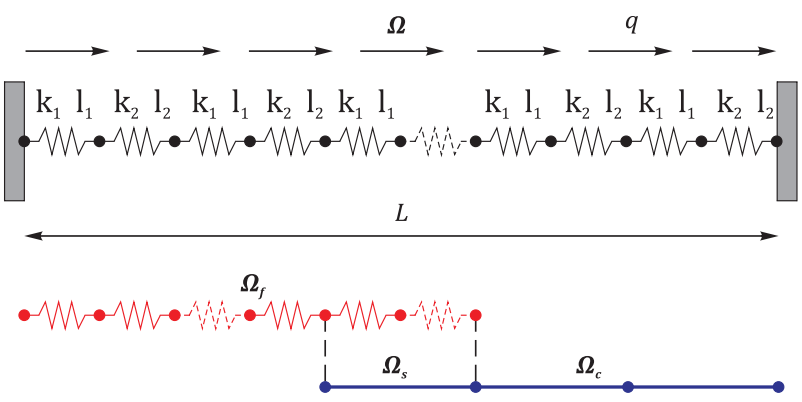

Figure 7. The Arlequin method in a particle model.

Table 4. Parameters of the particle-continuum model.

\begin{tabular}{cccccc}
\hline$k_{1}(\mathrm{~N} / \mathrm{m})$ & $k_{2}(\mathrm{~N} / \mathrm{m})$ & $l_{1}\left(10^{-4} \mathrm{~m}\right)$ & $l_{2}\left(10^{-4} \mathrm{~m}\right)$ & $I_{c}\left(10^{-1} \mathrm{~m}\right)$ & $L(\mathrm{~m})$ \\
\hline 100 & 1 & 1.5625 & 1.5625 & 1.25 & 3 \\
\hline
\end{tabular}

zone. The relative errors at selected points together with the maximum relative error in the whole domain can represent the responses of the whole model.

The maximum range of the coupling parameters $\alpha_{c}, \beta_{c}, \ell$, and $L_{s}$ are respectively $[0,1],[0,1],[0, L]$, and $\left[2 l_{c}, 20 l_{c}\right]$. The parameters are assumed to be statistically independent and uniformly distributed. Among others, $L_{s}$ is a discrete variable with values equal to $2 l_{c}, 4 l_{c}, 6 l_{c} \cdots 20 l_{c}$, where each value is equally likely. The sample size for each case is denoted by $N_{d}$. The convergence of Sobol' indices with respect to the sample sizes is presented in Appendix A.

\subsection{The coarse-fine bar model}

A both-ends clamped bar with uniform axial force $q=$ $1 \mathrm{~N} / \mathrm{m}$ is presented in Figure 4. The domain $\Omega$ is split into 
Table 5. The Sobol' indices of case 1 and case 2 for the particle-continuum model.

\begin{tabular}{|c|c|c|c|c|c|c|c|c|c|}
\hline & & \multicolumn{2}{|c|}{$E r r_{w}$} & \multicolumn{2}{|c|}{$\mathrm{Err}_{f}$} & \multicolumn{2}{|c|}{$\mathrm{Err}_{\mathrm{s}}$} & \multicolumn{2}{|c|}{$\mathrm{Err}_{c}$} \\
\hline & & $S_{i}$ & $S_{T i}$ & $S_{i}$ & $S_{T i}$ & $S_{i}$ & $S_{T i}$ & $S_{i}$ & $S_{T i}$ \\
\hline \multirow[t]{4}{*}{ case 1} & $\alpha_{c}$ & 0.7109 & 0.9321 & 0.9497 & 0.9935 & 0.9413 & 0.9836 & 0.9497 & 0.9936 \\
\hline & $\beta_{c}$ & 0 & 0 & 0 & 0 & 0 & 0 & 0 & 0 \\
\hline & $\ell$ & 0.0305 & 0.0958 & 0.0065 & 0.0503 & 0.0164 & 0.0587 & 0.0064 & 0.0503 \\
\hline & $L_{s}$ & 0.0254 & 0.2280 & 0 & 0 & 0 & 0 & 0 & 0 \\
\hline \multirow[t]{4}{*}{ case 2} & $\alpha_{c}$ & 0.0031 & 0.0031 & 0.0065 & 0.2872 & 0 & 0.0089 & 0.0084 & 0.3044 \\
\hline & $\beta_{c}$ & 0 & 0 & 0 & 0 & 0 & 0 & 0 & 0 \\
\hline & $\ell$ & 0.3496 & 0.6716 & 0.4524 & 0.9876 & 0.7212 & 0.9794 & 0.4196 & 0.9916 \\
\hline & $L_{s}$ & 0.3253 & 0.6474 & 0.0059 & 0.3272 & 0.0206 & 0.2707 & 0 & 0.3496 \\
\hline
\end{tabular}
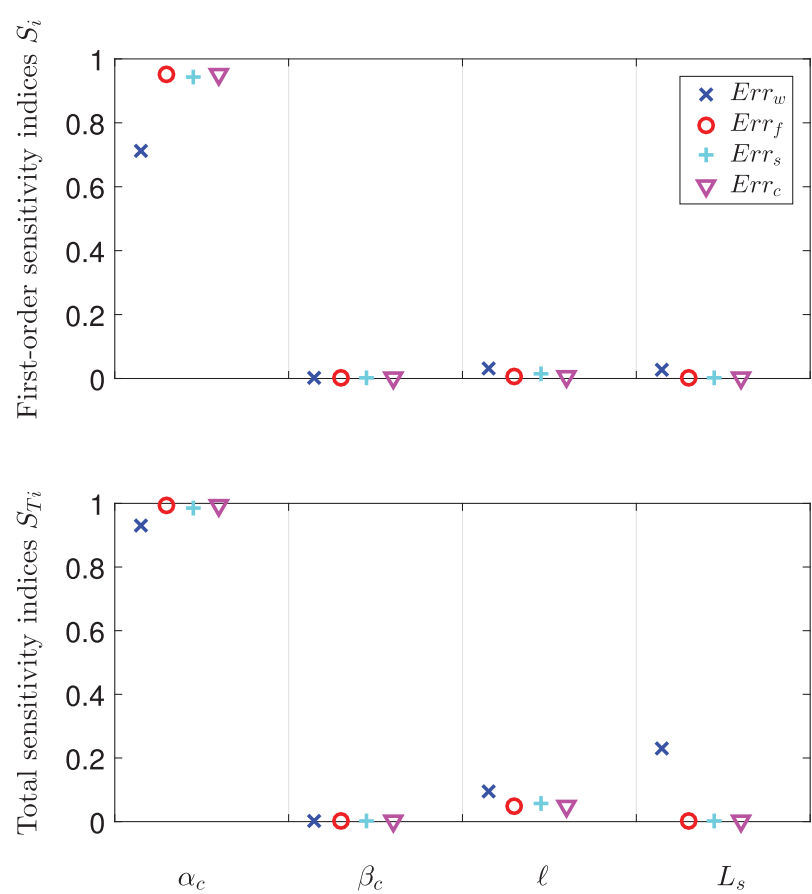

(a)
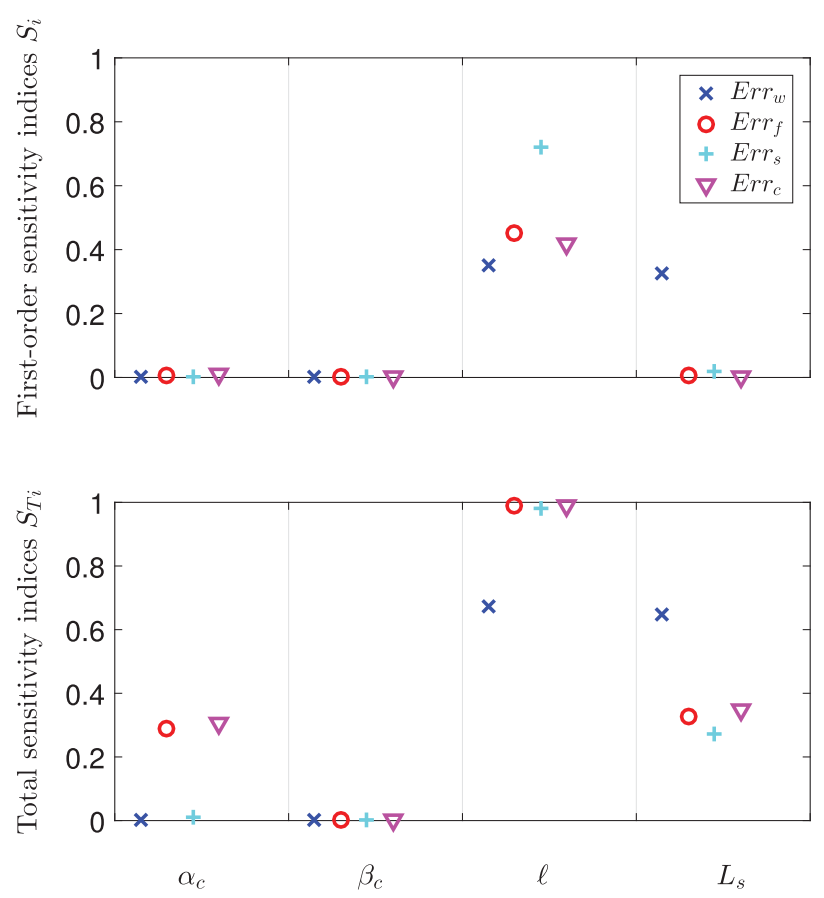

(b)

Figure 8. The Sobol' indices of (a) case-1 and (b) case-2 for the particle-continuum model. two sub-domains, where $\Omega_{f}$ is discretized by using a fine mesh, whilst a coarse mesh is considered for $\Omega_{c}$. The coupling between these two models is achieved in the overlapping zone by the Arlequin method with the $H^{1}$-type coupling operator.

The geometrical and material parameters are illustrated in Table 1, where $E$ and $q$ represent the Young's modulus and the uniform axial force respectively, $l_{c}$ and $l_{f}$ stands for the element length of the coarse model and the fine model, respectively. The cross-sectional area is set to unity for simplicity.

A linear Lagrange interpolation is used for the discretization of the displacement fields and the Lagrange multipliers:

$$
\langle N\rangle=\left\langle\frac{1-\xi}{2} \frac{1+\xi}{2}\right\rangle
$$

where $\xi$ represents the natural local coordinate. The bar is isotropic and meets Hook's law. The axial strain and stress can be expressed as follows:

$$
\sigma_{x}=E \varepsilon_{x} \quad \text { and } \quad \varepsilon_{x}=\left\langle\boldsymbol{N}_{, x}\right\rangle\left\{\boldsymbol{q}_{e}\right\}
$$

where $\boldsymbol{q}_{e}$ denotes the elementary displacement vector.

Lagrange multipliers share the same mesh with the displacement in the coarse model. Considering Eqs. (1), (3), (4), (31), and (30), a linear system is obtained as shown in Eq. (5). For a given set of input parameters, solving this linear system results in the solution of each sub-domain. And then the displacement field $\boldsymbol{U}$ of the multi-scale model are calculated via Eq. (6). For this example, the analytic solution can be obtained easily, which will be used as the reference $\boldsymbol{U}_{\text {ref }}$. For brevity, here we do not present the details on how to obtain the analytic solution.

Now we draw a sample of size $N_{d}=2^{10}$ in the maximum ranges of the coupling parameters $\left\langle\alpha_{c}, \beta_{c}, \ell, L_{s}\right\rangle$ (see Table 2 case 1). Note that $N_{d}=2^{10}$ is sufficient to guarantee the convergence of GSA for this example. After building the SPCE model between the coupling parameters and the relative errors, Sobol' indices are calculated. The resulting firstorder and total Sobol' indices are displayed in Figure 5(a) and Table 3 case 1 . We recall that, in GSA, the first-order sensitivity index $S_{i}$ evaluates the sensitivity due to the input variable $x_{i}$ alone, while the total sensitivity index $S_{T i}$ measures the total effect caused by $x_{i}$ including the interactions. The higher the sensitivity index is, the more important $x_{i}$ is for the model response. Apparently, $1 \geqslant S_{T i} \geqslant S_{i} \geqslant 0$. We can notice that the most influential parameter is the internal energy partition function $\alpha_{c}$, whose total sensitivity indices 

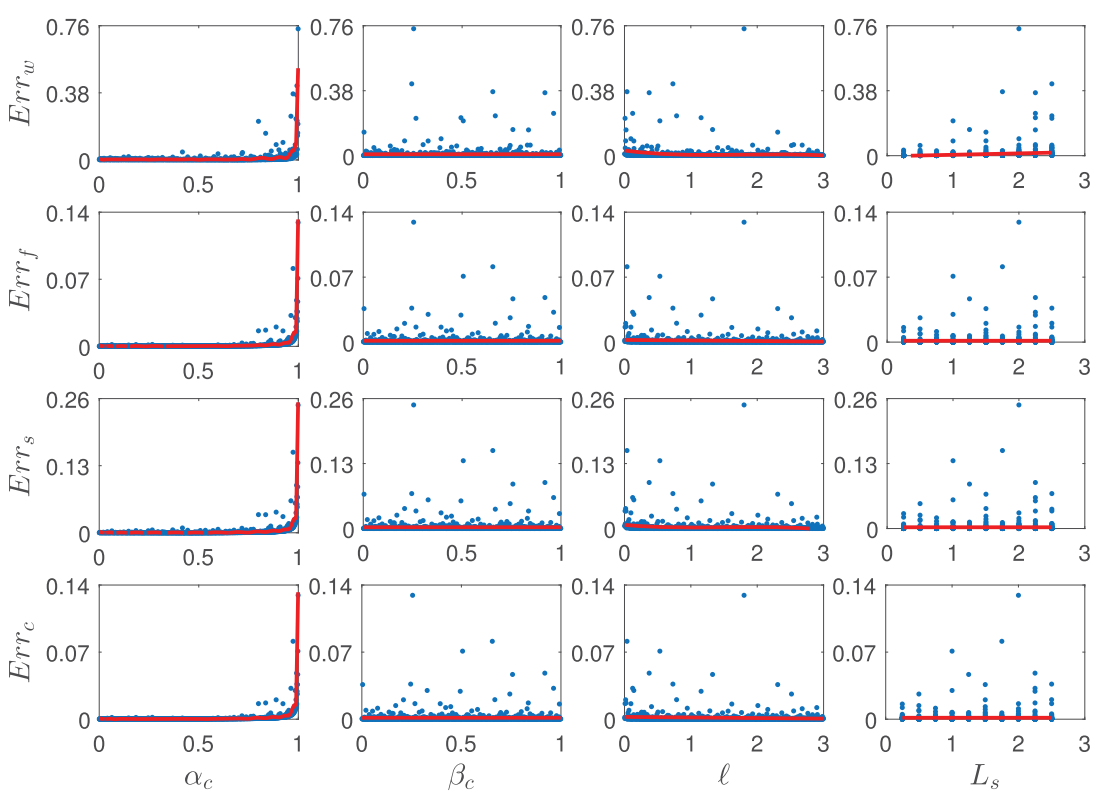

(a)
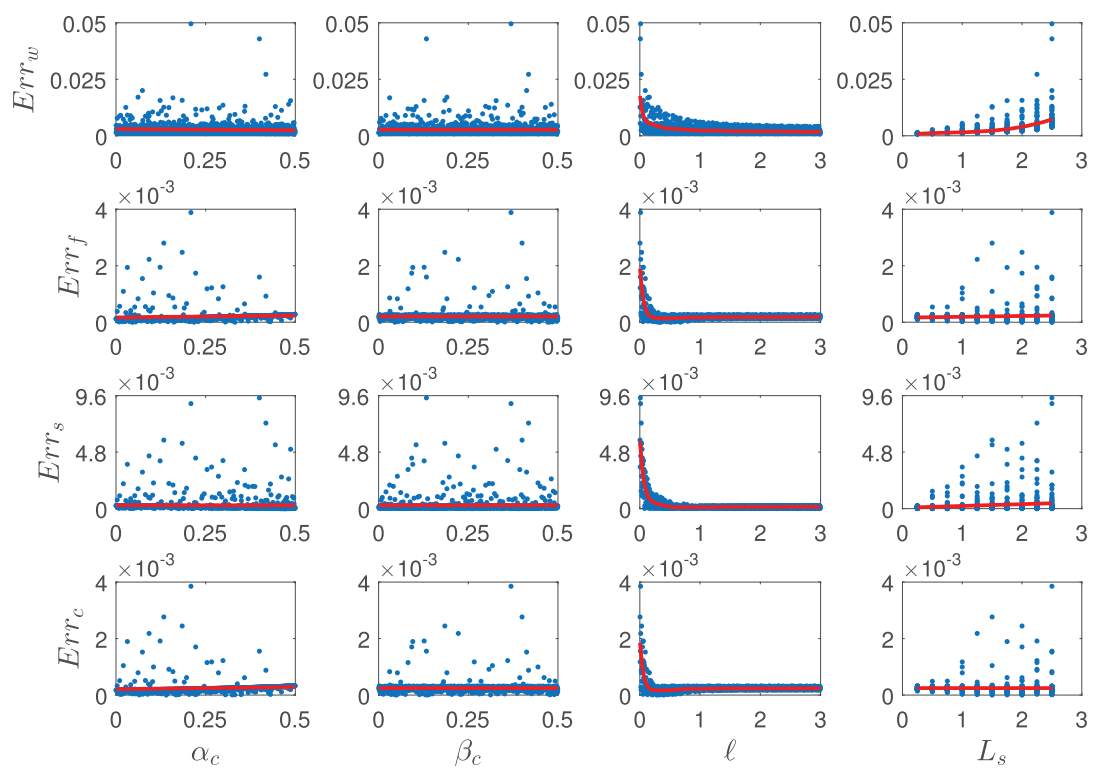

(b)

Figure 9. The marginal effect of Arlequin parameters in (a) case 1 and (b) case 2 for the particle-continuum model.

for the four model responses $\operatorname{Err}_{j}(j=w, f, s, c)$ are all higher than 0.93 (very close to 1). In Figure 6(a), we plot all the obtained data of $\operatorname{Err}_{j}(j=w, f, s, c)$ versus the coupling parameters $\left\langle\alpha_{c}, \beta_{c}, \ell, L_{s}\right\rangle$. The curves in Figure 6(a) represent the marginal effect of each parameter on different model responses. It is worth noting that when $\alpha_{c}$ approaches 1 , the relative errors raise sharply. Especially the maximum relative error of the whole domain $\mathrm{Err}_{w}$ can increase to about 50\%, which indicates that the effectiveness of the coupling drops drastically. Therefore the value of $\alpha_{c}$ should be far from 1 , that is to say, the internal energy distribution should be always partially shared with fine model. This is consistent with the results in Ben Dhia et al. [8]. It is reasonable since the fine model describes the energy of the domain more accurately and it deserves a higher weight.
From Figure 6(a) and Table 3 case 1, we notice that the Sobol' indices of the external energy partition function $\beta_{c}$ are equal to 0 , which means that $\beta_{c}$ does not influence the accuracy of the coupled model. The state-of-the art about the Arlequin method mainly discuss the choice of $\alpha_{c}$, while the value of $\beta_{c}$ is set equal to $\alpha_{c}$ by default, such as in Ben Dhia et al. [6] and Guidault and Belytschko [37].

The Sobol' indices of the characteristic length $\ell$ and the size of the coupling zone $L_{s}$ are very low for this example. The total Sobol' index of $L_{s}$ on $\operatorname{Err}_{w}\left(S_{T i}=0.2270\right)$ is higher than the corresponding first-order Sobol' index $\left(S_{i}=0.0258\right)$, which means the effect of $L_{s}$ on the coupling is mainly reflected in interactions. Besides, in Figure 6(a), the marginal effect curves of model responses versus the two coupling parameters $\left\langle\ell, L_{s}\right\rangle$ show very slight changes. The 


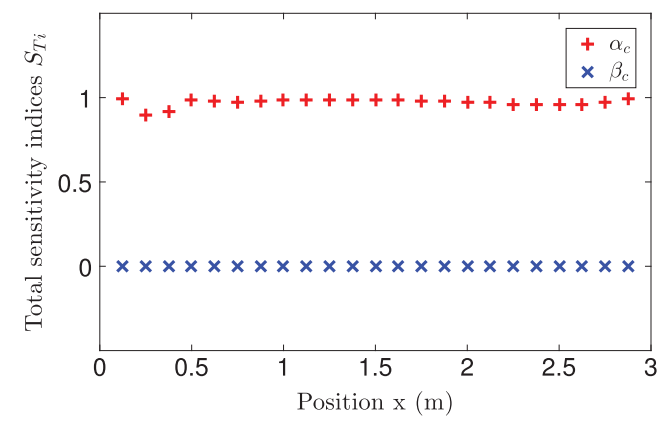

Figure 10. The total Sobol' indices of $\alpha_{c}$ and $\beta_{c}$ at different points in the domain of the particle-continuum model.
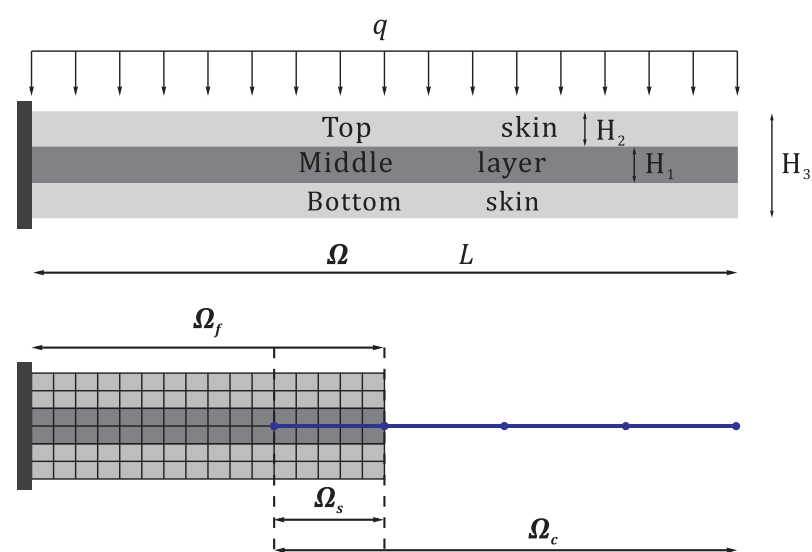

Figure 11. Sketch of a cantilever sandwich beam.

results above indicate that the sensitivity of the coupling due to $\ell$ and $L_{s}$ is very low compared to $\alpha_{c}$ : these two parameters slightly affect the coupling in this case.

To further investigate the influence of $\ell$ and $L_{s}$, we narrow the sampling range of energy partition functions $\alpha_{c}, \beta_{c}$ from $[0,1]$ to $[0,0.5]$, while the sampling range of $\ell$ and $L_{s}$ stay the same (see Table 2 case 2). We draw samples of size $N_{d}=2^{10}$ from the new prior distribution of case 2 followed by numerical computations to obtain the relative errors. Subsequently, a GSA is performed based on the Bayesian SPCE. The obtained Sobol' indices are presented by Figure 5(b) and Table 3 case 2. The sensitivity indices of $\alpha_{c}$ on the maximum relative error $E r r_{w}$ decrease drastically from a high level in case 1 $\left(S_{i}=0.7126, S_{T i}=0.9318\right)$ to almost $0\left(S_{i}=S_{T i}=0.0027\right)$. Besides, the value of the maximum relative error $E r r_{w}$ also decreases significantly. Here the maximum relative error is less than $5 \%$, which is much less than about $50 \%$ in case 1 . This implies that when the sampling range of energy partition functions reduce to $[0,0.5]$, the coupling is slightly sensitive to $\alpha_{c}$ and the accuracy of the coupled model is significantly improved. The total Sobol' indices of $\alpha_{c}$ on $E r r_{f}$ and $E r r_{c}$ are obviously higher than the corresponding first-order Sobol' indices, while all the Sobol' indices of $\alpha_{c}$ on $E r r_{s}$ are very low. That means, in this case, the effect of the choice of $\alpha_{c}$ mainly appears in the whole domain except $\Omega_{s}$ in the form of interactions and are very small in the coupling zone $\Omega_{s}$. The marginal effect of $\alpha_{c}$ on model responses is negligible as illustrated in Figure 6(b). In particular, the maximum relative error of the displacement in the whole domain has been reduced to an acceptable level. We can also
Table 6. Parameters of the 2D-1D sandwich FE models.

\begin{tabular}{lcccccc}
\hline$E_{1}(\mathrm{MPa})$ & $E_{2}(\mathrm{MPa})$ & $L(\mathrm{~m})$ & $H_{3}(\mathrm{~m})$ & $H_{1} / H_{2}$ & $\mu_{1}$ & $\mu_{2}$ \\
\hline 6.9 & $6.9 \times 10^{4}$ & 0.2 & 0.01 & 1 & 0.3 & 0.3 \\
\hline
\end{tabular}

Table 7. The Sobol' indices of case 1 and case 2 for the 2D-1D sandwich model.

\begin{tabular}{|c|c|c|c|c|c|c|c|c|c|}
\hline & \multicolumn{2}{|c|}{$E r r_{w}$} & \multicolumn{2}{|c|}{$E r r_{f}$} & \multicolumn{2}{|c|}{$E r r_{s}$} & \multicolumn{2}{|c|}{$E r r_{c}$} \\
\hline & & $S_{i}$ & $S_{T i}$ & $S_{i}$ & $S_{T i}$ & $S_{i}$ & $S_{T i}$ & $S_{i}$ & $S_{T i}$ \\
\hline \multirow[t]{4}{*}{ case 1} & $\alpha_{c}$ & 0.4621 & 0.9221 & 0.6572 & 0.9598 & 0.5417 & 0.9510 & 0.6828 & 0.9674 \\
\hline & $\beta_{c}$ & 0 & 0 & 0 & 0 & 0 & 0 & & 0 \\
\hline & $\ell$ & 0.0067 & 0.1215 & 0.0111 & 0.0926 & 0.0056 & 0.0494 & 0.0088 & 0.0771 \\
\hline & $L_{s}$ & 0.0713 & 0.4468 & 0.0291 & 0.2564 & 0.0434 & 0.4341 & 0.0238 & 0.2452 \\
\hline \multirow[t]{4}{*}{ case 2} & $\alpha_{c}$ & 0.0090 & 0.5852 & 0.3018 & 0.6475 & 0.1622 & 0.6051 & 0.2180 & 0.7003 \\
\hline & $\beta_{c}$ & 0 & 0 & 0 & 0 & 0 & 0 & 0 & 0 \\
\hline & $\ell$ & 0 & 0 & 0.0710 & 0.2413 & 0.0987 & 0.3313 & 0.0952 & 0.6043 \\
\hline & $L_{s}$ & 0.4148 & 0.9910 & 0.2411 & 0.5636 & 0.2185 & 0.6717 & 0.0367 & 0.5669 \\
\hline
\end{tabular}

note that the influence of $\beta_{c}$ still seems to be negligible as the Sobol' indices of $\beta_{c}$ are still equal to 0 . Hence, choosing the value of energy partition functions in the range of $[0,0.5]$ is feasible when using the Arlequin method.

In case 2, the Sobol' indices shown in Figure 5(b) and Table 3 case 2 demonstrate that the coupling accuracy is mainly affected by the characteristic length $\ell$ and the size of the coupling zone $L_{s}$, as the Sobol' indices rise significantly compared to case 1 . The interactions of these factors are evident as $S_{T i}>S_{i}$. From the curves displayed in Figure 6(b), we can see that, the closer the value of $\ell$ to 0 , the greater the model responses. When $\ell=0, H^{1}$ coupling operator is converted to $L^{2}$ coupling operator. The increase of relative errors at $\ell=0$ implies that $H^{1}$ coupling operator is more reliable than $L^{2}$ coupling operator. The same conclusion has been reported in Ben Dhia et al. [8] and Guidault and Belytschko [37]. Considering Eq. (3), the existence of gradients coupling terms can reduce the condition number of stiffness matrix which leads to better accuracy and stability of the coupling. The characteristic length $\ell$ is used to balance the two kinds of coupling conditions. Therefore, according to the marginal effect of $\ell$ in Figure 6(b), $\ell>2 l_{c}$ is suggested for the coupling.

From Figure 6(b), we can notice that as the size of the coupling zone $L_{s}$ increases, the model responses change slowly. In particular, there is a slightly positive correlation between maximum relative error $E r r_{w}$ and $L_{s}$. Besides, considering the lower degrees of freedom, it is suggested that a relatively smaller coupling size should be adopted. We suggest that $L_{s}$ in $\left[2 l_{c}, 6 l_{c}\right]$ is appropriate.

\subsection{The particle-continuum model}

A particle model with uniform axial force $q=1 \mathrm{~N} / \mathrm{m}$ is illustrated in Figure 7, where the connections between these particles are represented by periodically distributed springs with stiffness $k_{1}, k_{2}$ and equilibrium length $l_{1}, l_{2}$.

To reduce the size of the particle model, a particle-continuum model that involves the particle model in $\Omega_{f}$ and a continuum model in $\Omega_{c}$ is considered. The Arlequin method is used to couple the two different models. As suggested in [9], a linear elastic bar model is employed as the continuum model, whose modulus $E$ can be calculated as: 

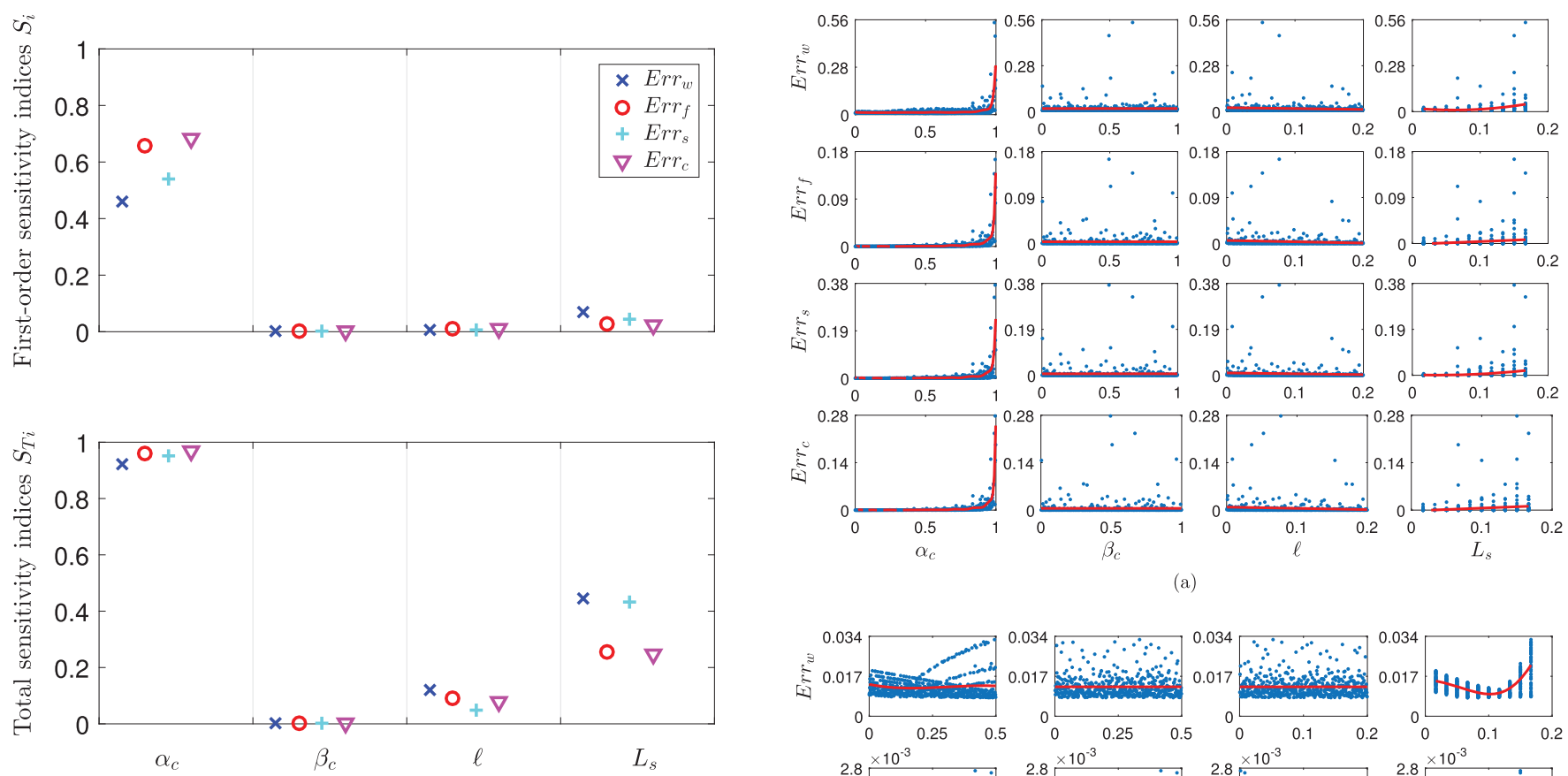

(a)
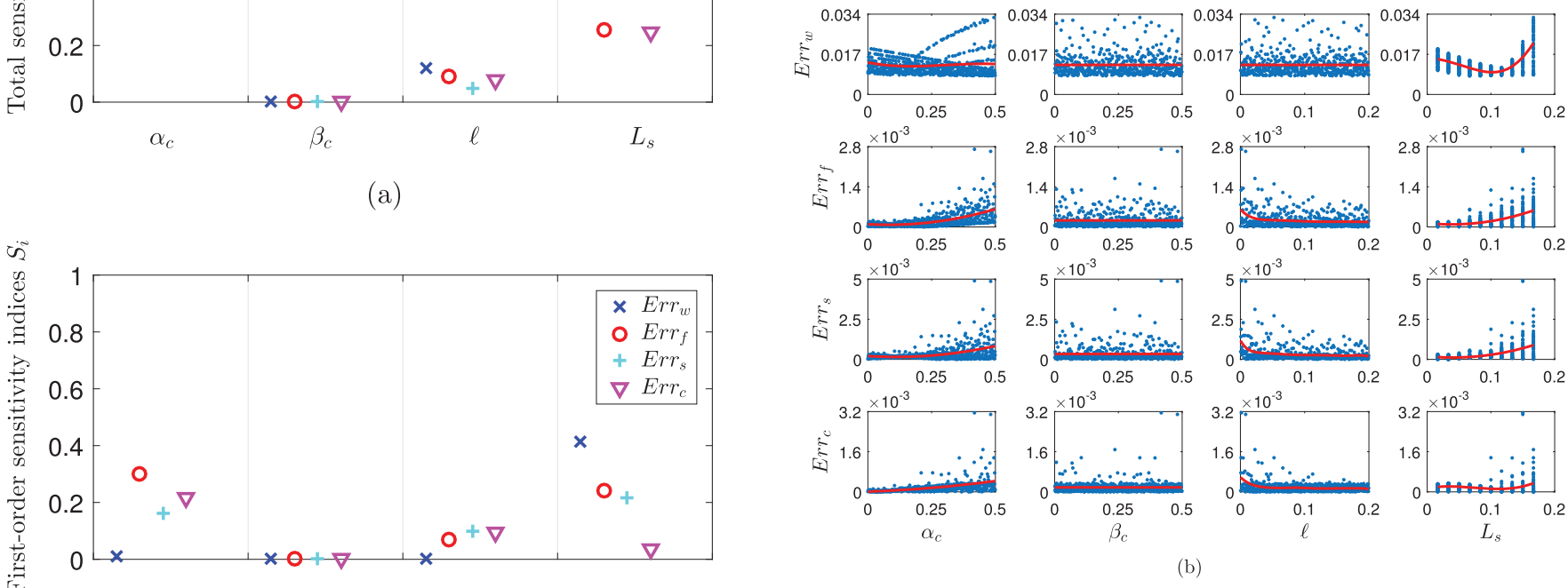

(b)

Figure 13. The marginal effect of Arlequin parameters in (a) case 1 and (b) case 2 for the 2D-1D sandwich model.

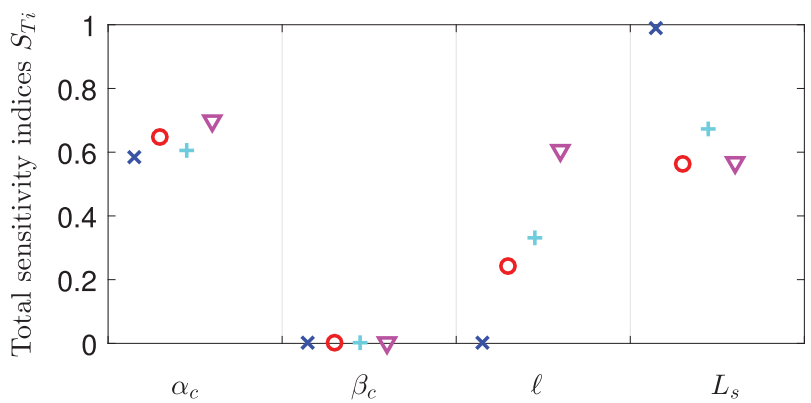

multipliers. Based on the formulations in Section 2, one can easily obtain a linear system Eq. (5) and consequently the global displacement field via Eq. (6). The solution of a full particle model is adopted as the reference $\boldsymbol{U}_{\text {ref }}$.

Same as the previous example, we investigate the sensitivity of the coupling parameters in their maximum sampling range (see Table 2 case 1 ). Note that the sample size $N_{d}$ for this example is set to $2^{10}$ which can ensure the convergence of GSA. The Sobol' indices and the marginal effect shown in Table 5 case 1, Figures $8(a)$ and 9(a) illustrate a similar trend as that in the coarse-fine bar model. The internal energy partition $\alpha_{c}$ is the most influential parameter. More weight should be attached to the particle model than the continuum model. According to the Sobol' indices, the external energy partition $\beta_{c}$ has no effect on the coupling accuracy. For further investigation, we select a series of uniformly distributed points in the whole domain with interval equal to $l_{c}$ and study the effects of the energy partition functions on the relative error at these points. The total Sobol' indices of each point are illustrated in Figure 10. The results show that the Sobol' indices of $\alpha_{c}$ are very high in the whole domain, which indicates the huge impact of $\alpha_{c}$ on the cou-

pling. The Sobol' indices of $\beta_{c}$ are equal to 0 in the whole ization of the displacement fields and the Lagrange

Note that the cross-sectional area of the bar is set to be unity for simplicity.

The parameters of the particle-continuum model are listed in Table 4 , where $l_{c}$ represents the elementary length of the continuum model. 
domain, which means that $\beta_{c}$ does not influence the coupling accuracy in this case.

Then a narrower sampling range $[0,0.5]$ is considered to reduce the influence of $\alpha_{c}$ and to further investigate the influence of $\ell$ and $L_{s}$ (see Table 2 case 2). In Figures 8(b) and $9(\mathrm{~b})$, the same phenomenon has been observed as in the previous example: the sensitivity indices of $\alpha_{c}$ and the maximum relative error $\operatorname{Err}_{w}$ both decrease significantly and all the model responses are reduced to an acceptable level (the maximum relative error is less than 5\%). The results show that the suitable range of the energy partition functions lies in $[0,0.5]$.

In case 2 with optimal choice of energy partition functions, the Sobol' indices in Figure 8(b) and Table 5 case 2 show that $\ell$ and $L_{s}$ are the main influencing factors for the coupling. In Figure 9(b), the marginal effect curves show that a smaller $\ell$ close to 0 leads to a higher relative error. Similar results have been reported by Guidault and Belytschko [23], which indicate that the $H^{1}$ coupling may be beneficial to couple a very coarse mesh with an atomistic model than $L^{2}$ coupling where $\ell=0$. To achieve a good coupling, the characteristic length $\ell$ is suggested to be larger than $2 l_{c}$.

According to the marginal effect of $L_{s}$ in Figure 9(b), the maximum relative error increases with the increase of $L_{s}$. Thus, a small coupling zone $L_{s}$ in the range $\left[2 l_{c}, 6 l_{c}\right]$ is recommended.

\subsection{The 2D-1D sandwich model}

In this part, a cantilever sandwich beam subjected to a uniformly distributed load $q=100 \mathrm{~N} / \mathrm{m}$ is considered, as shown in Figure 11. The geometric and material parameters of the sandwich beam are listed in Table 6, where $E$, $\mu$, and $H$ stand for Young's modulus, Poisson's ratio, and thickness, respectively. The top and bottom layers of the beam have the same geometric and material parameters. The subscript 1 and 2 in Table 6 represent the parameters related to the middle layer and top layers, respectively. The thickness of the whole beam is $H_{3}$.

The 2D-1D sandwich multi-scale model developed in [25] is used to model this beam, in which the $2 \mathrm{D}$ model is used near the boundary to accurately capture the boundary effects and the 1D sandwich model in the rest region to reduce the computational cost. The detailed formulations of the $2 \mathrm{D}-1 \mathrm{D}$ coupling model are presented in Appendix B. In the thickness direction, six $2 \mathrm{D}$ elements are sufficient. In the longitudinal direction, the elementary length of the 2D FE model and $1 \mathrm{D}$ beam model are, respectively, $l_{f}=$ $1.67 \times 10^{-3} \mathrm{~m}$ and $l_{c}=8.33 \times 10^{-3} \mathrm{~m}$. The mesh scheme considered here is sufficient to yield convergent results. Reference solution $\boldsymbol{U}_{\text {ref }}$ is the middle plane displacement fields obtained by using the $2 \mathrm{D}$ model in the whole structure.

Similar to the previous two examples, we analyze two cases as shown in Table 2. The difference consists in using two different ranges of weight functions $\alpha_{c}$ and $\beta_{c}$. The sample size $N_{d}$ for this example is set to $2^{9}$ which can ensure

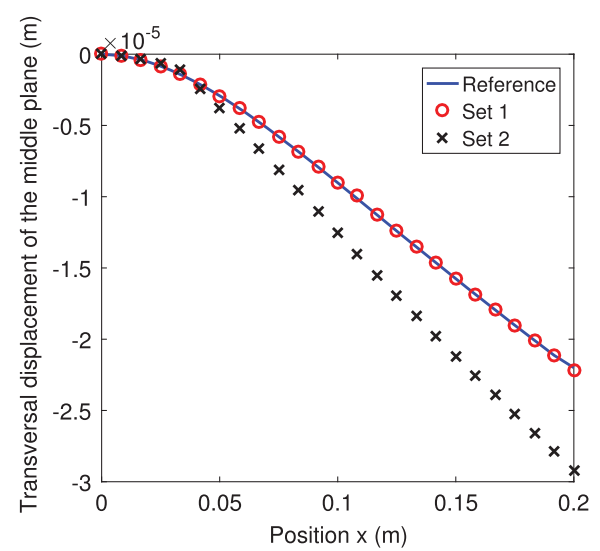

Figure 14. Transverse displacement along the beam axis.

the convergence of GSA. The Sobol' indices are presented in Table 7 and plotted in Figure 12 for clarity. The marginal effect of each parameter on different model responses is shown in Figure 13. The following conclusions could be found based on the obtained results:

- Similar to the previous two examples, $\alpha_{c}$ is still the most important parameter, that is, the model responses increase sharply when $\alpha_{c}$ approaches to 1 as shown in Figure $13(\mathrm{a})$. When $\alpha_{c}$ is in the range $[0,0.5]$, the effect of $\alpha_{c}$ is significantly reduced compared to case 1 . In case 2, with the increase of $\alpha_{c}$, the maximum relative error $\operatorname{Err}_{w}$ remains stable, but the relative errors of the three representative points $E r r_{f}, E r r_{s}$, and $\operatorname{Err}_{c}$ increase slowly. It means that the coupling effects of the whole domain may become worse. This further verifies that the coarse model should be associated with less weight in this heterogeneous structure. Considering $E r r_{w}$ has been reduced to an acceptable range $\left(E r r_{w}<3.4 \%\right)$ in case 2, the range of $[0,0.5]$ is desirable for the energy weight functions.

- The Sobol' indices of $\beta_{c}$ are still equal to 0 in both cases, which indicates that $\beta_{c}$ still has negligible effect.

- In case 1 , the characteristic length $\ell$ slightly affects the coupling solution. The effect of $\ell$ mainly appears in the form of interactions as $S_{T i}>S_{i} \approx 0$. For case $2, \mathcal{L}$ has no effect on the maximum relative error $\operatorname{Err}_{w}$ as $S_{T i}=S_{i}=0$, but affects the coupling of the selected points in the domain. This result can also be observed from the marginal effect curves in Figure 13(b), where $E r r_{w}$ does not change with the increase of $\ell$, while $E r r_{f}$, $E r r_{s}$, and $E r r_{c}$ increase when $\ell$ is approaching 0. One could also note that $\ell=0$ would lower the coupling accuracy, which coincides with the conclusion drawn by $\mathrm{Hu}$ et al. [25]. Here, we suggest that $\mathcal{L}$ should be greater than $2 l_{c}$ to achieve a good coupling solution.

- The size of the coupling zone $L_{s}$ is the second most influential factor in this example. For both cases, with the increase of $L_{s}, E r r_{w}$ decreases slightly and then increases significantly, while $\operatorname{Err}_{f}, \operatorname{Err}_{s}$, and $\operatorname{Err}_{c}$ increase slowly. This indicates that a large coupling zone may deteriorate the precision of coupling and cause unsatisfactory solution in the whole domain. Like the previous 
two examples in Sections 4.1 and 4.2, we can see that taking the value of $L_{s}$ in the range of $\left[2 l_{c}, 6 l_{c}\right]$ is still reasonable.

To verify the above findings, we select two sets of coupling parameters: (a) set 1: $\alpha_{c}=0.01, \beta_{c}=0.5, \ell=3 l_{c}$, $L_{s}=2 l_{c}$, (b) set 2: $\alpha_{c}=0.99, \beta_{c}=0.5, \ell=l_{c}, L_{s}=16 l_{c}$. In set 1 , all coupling parameters are within the suggested ranges, while in set 2 , values of $\alpha_{c}, \ell$, and $L_{s}$ are beyond the suggested ranges. We present the transversal displacements of the middle plane of the sandwich structure in Figure 14 by using these two sets of parameters. The reference solution of displacement obtained by using the full $2 \mathrm{D}$ model is also plotted and compared to the coupled results. One can easily find that the results in set 1 agree well with the reference solution, whereas results obtained by set 2 deflect far from the reference. This proves that the suggested ranges of the coupling parameters are reliable. It also verifies that the Arlequin method, with reasonable coupling parameters, can yield accurate solutions. On the other hand, if the coupling parameters are not well set, the model errors can be relatively large, leading to inaccurate solutions.

\section{Conclusions}

In this article, a data-driven approach based on SPCE is used to quantitatively and qualitatively investigate the influence of Arlequin parameters, namely the energy partition functions $\alpha_{c}$ and $\beta_{c}$, the characteristic length $\ell$ of the coupling operator and the size of the coupling zone $L_{s}$, on the accuracy of multi-scale models. Three typical benchmark tests are considered, which are a coarse-fine bar model, a particle-continuum model and a 2D-1D sandwich model. The influence of each parameter and their interactions are carefully discussed and the optional settings of these factors can be drawn as follows:

1. The internal energy partition function is the most important factor in the coupling and should be put on the refined model. The optimal range of $\alpha_{c}$ is $[0,0.5]$.

2. According to the results, the external energy partition function $\beta_{c}$ hardly affects the coupling accuracy and the influence of $\beta_{c}$ can be almost ignored compared to that of $\alpha_{c}$. For simplicity, we suggest that the value of $\beta_{c}$ is set equal to $\alpha_{c}$.

3. The characteristic length $\ell$ of the coupling operator is not supposed to be small. We suggest $\ell>2 l_{c}$.

4. The size of the coupling zone $L_{s}$ should not be large. The recommended range of $L_{s}$ is $\left[2 l_{c}, 6 l_{c}\right]$.

The multi-scale models analyzed in this work are all linear models. The Arlequin parameters in cases of non-linear multi-scale models $[12,15,39]$ will be analyzed by the proposed approach in subsequent work. Besides, the location of the coupling region is intentionally arranged in the middle of the entire domain, which deserves further investigation. The data-driven strategy presented in this work shows huge potential for analyzing complicated mechanical problems.

\section{Funding}

This work has been supported by the National Natural Science Foundation of China (Grant Nos.: 11702199 and 11772238) and the Natural Science Foundation of Hubei Province (Grant No: 2017CFB147). The authors would like to acknowledge the support of EU H2020 COMPOSELECTOR project (Grant No: 721105).

\section{ORCID}

Jian Liu (D) http://orcid.org/0000-0002-4380-3300

\section{References}

[1] J. Q. Broughton, F. F. Abraham, N. Bernstein, and E. Kaxiras, "Concurrent coupling of length scales: Methodology and application,” Phys. Rev. B, vol. 60, no. 4, pp. 2391-2403, 1999.

[2] G. J. Wagner and W. K. Liu, "Coupling of atomistic and continuum simulations using a bridging scale decomposition," J. Comput. Phys., vol. 190, no. 1, pp. 249-274, 2003.

[3] S. P. Xiao and T. Belytschko, "A bridging domain method for coupling continua with molecular dynamics," Comput. Methods Appl. Mech. Eng., vol. 193, no. 17, pp. 1645-1669, 2004.

[4] H. Ben Dhia, "Multiscale mechanical problems: the arlequin method," C. R. Acad. Sci. 2B Mech. Phys. Astron., vol. 326, no. 12, pp. 899-904, 1998.

[5] H. Ben Dhia, Numerical modelling of multiscale problems: the arlequin method, in: CD Proceedings of ECCM'99, Munchen, 1999.

[6] H. Ben Dhia and G. Rateau, "The arlequin method as a flexible engineering design tool,” Int. J. Numer. Methods Eng., vol. 62, no. 11, pp. 1442-1462, 2005.

[7] H. Ben Dhia, "Global-local approaches: the arlequin framework,” Eur. J. Comput. Mech., vol. 15, no. 1-3, pp. 67-80, 2006.

[8] H. Ben Dhia, "Further insights by theoretical investigations of the multiscale arlequin method," Int. J. Multiscale Comput. Eng., vol. 6, no. 3, pp. 215-232, 2008.

[9] P. T. Bauman, H. Ben Dhia, N. Elkhodja, J. T. Oden, and S. Prudhomme, "On the application of the arlequin method to the coupling of particle and continuum models," Comput. Mech., vol. 42, no. 4, pp. 511-530, 2008.

[10] S. Prudhomme, H. Ben Dhia, P. T. Bauman, N. Elkhodja, and J. T. Oden, "Computational analysis of modeling error for the coupling of particle and continuum models by the arlequin method," Comput. Methods Appl. Mech. Eng., vol. 197, no. 4142, pp. 3399-3409, 2008.

[11] S. Prudhomme, R. Bouclier, L. Chamoin, H. Ben Dhia, and J. T. Oden, "Analysis of an averaging operator for atomic-to-continuum coupling methods by the Arlequin approach," in Numerical Analysis of Multiscale Computations, B. Engquist, O. Runborg, Y.H. R. Tsai (Eds.), Springer, Berlin, pp. 369-400, 2012.

[12] H. Hu, S. Belouettar, M. Potier-Ferry, E. M. Daya, and A Makradi, "Multi-scale nonlinear modelling of sandwich structures using the arlequin method," Compos. Struct., vol. 92, no. 2, pp. 515-522, 2010.

[13] H. Hu, N. Damil, and M. Potier-Ferry, "A bridging technique to analyze the influence of boundary conditions on instability patterns," J. Comput. Phys., vol. 230, no. 10, pp. 3753-3764, 2011.

[14] K. Yu, H. Hu, S. Chen, S. Belouettar, and M. Potier-Ferry, "Multi-scale techniques to analyze instabilities in sandwich structures," Compos. Struct., vol. 96, no. 2, pp. 751-762, 2013.

[15] Q. Huang, Y. Liu, H. Hu, Q. Shao, K. Yu, G. Giunta, S. Belouettar, and M. Potier-Ferry, "A fourier-related double scale analysis on the instability phenomena of sandwich plates," Comput. Methods Appl. Mech. Eng., vol. 318, pp. 270-295, 2017. 
[16] Q. Z. He, H. Hu, S. Belouettar, G. Giunta, K. Yu, Y. Liu, F. Biscani, E. Carrera, and M. Potier-Ferry, "Multi-scale modelling of sandwich structures using hierarchical kinematics," Compos. Struct., vol. 93, no. 9, pp. 2375-2383, 2011.

[17] F. Biscani, G. Giunta, S. Belouettar, E. Carrera, and H. Hu, "Variable kinematic plate elements coupled via arlequin method," Int. J. Num. Methods Eng., vol. 93, no. 2, pp. 697-708, 2011.

[18] F. Biscani, G. Giunta, S. Belouettar, H. Hu, and E. Carrera, "Mixed-dimensional modeling by means of solid and higherorder multi-layered plate finite elements," Mech. Adv. Mater. Struct., vol. 23, no. 9, pp. 960-970, 2016.

[19] S. Li, S. Roy, and V. Unnikrishnan, "Modeling of fracture behavior in polymer composites using concurrent multi-scale coupling approach," Mech. Adv. Mater. Struct. pp. 1-9, 2016.

[20] K. Kpogan, Y. Tampango, H. Zahrouni, M. Potier-Ferry, and H. Ben Dhia, "Computing flatness defects in sheet rolling by arlequin and asymptotic numerical methods," Key Eng. Mater., vol. 611, pp. 186-193, 2014.

[21] K. Kpogan, H. Zahrouni, M. Potier-Ferry, and H. B. Dhia, "Buckling of rolled thin sheets under residual stresses by ANM and arlequin method," Int. J. Mater. Form., vol. 10, no. 3, pp. 389-404, 2017.

[22] H. Qiao, Q. D. Yang, W. Q. Chen, and C. Z. Zhang, "Implementation of the arlequin method into ABAQUS: Basic formulations and applications," Adv. Eng. Softw., vol. 42, no. 4, pp. 197-207, 2011.

[23] P. Guidault and T. Belytschko, "Bridging domain methods for coupled atomistic-continuum models with $L^{2}$ or $H^{1}$ couplings," Int. J. Num. Methods Eng., vol. 77, no. 11, pp. 1566-1592, 2009.

[24] W. C. Sun and A. Mota, "A multiscale overlapped coupling formulation for large-deformation strain localization," Comput. Mech., vol. 54, no. 3, pp. 803-820, 2014.

[25] H. Hu, S. Belouettar, M. Potier-Ferry, and E. M. Daya, "Multiscale modelling of sandwich structures using the arlequin method part I: Linear modelling," Finite Elements Anal. Design, vol. 45, no. 1, pp. 37-51, 2008.

[26] B. Sudret, "Global sensitivity analysis using polynomial chaos expansions," Reliab. Eng. Syst. Saf., vol. 93, no. 7, pp. 964-979, 2008.

[27] A. Saltelli, M. Ratto, T. Andres, F. Campolongo, J. Cariboni, D. Gatelli, M. Saisana, and S. Tarantola, Global Sensitivity Analysis: The Primer. West Sussex, UK: Wiley, 2008.

[28] I. M. Sobol', "Sensitivity estimates for nonlinear mathematical models," Math. Modell. Comput. Exp., vol. 1, no. 4, pp. 407-414, 1993.

[29] G. Blatman and B. Sudret, "Sparse polynomial chaos expansions and adaptive stochastic finite elements using a regression approach," C. R. Mécanique, vol. 336, no. 6, pp. 518-523, 2008.

[30] G. Blatman and B. Sudret, "Efficient computation of global sensitivity indices using sparse polynomial chaos expansions," Reliab. Eng. Syst. Saf., vol. 95, no. 11, pp. 1216-1229, 2010.

[31] G. Blatman and B. Sudret, "An adaptive algorithm to build up sparse polynomial chaos expansions for stochastic finite element analysis,” Probab. Eng. Mech., vol. 25, no. 2, pp. 183-197, 2010.

[32] G. Blatman and B. Sudret, "Adaptive sparse polynomial chaos expansions based on least angle regression," J. Comput. Phys., vol. 230, no. 6, pp. 2345-2367, 2011.

[33] C. Hu and B. D. Youn, "Adaptive-sparse polynomial chaos expansion for reliability analysis and design of complex engineering systems," Struct. Multidiscip. Optim., vol. 43, no. 3, pp. 419-442, 2011.

[34] N. Fajraoui, T. A. Mara, A. Younes, and R. Bouhlila, "Reactive transport parameter estimation and global sensitivity analysis using sparse polynomial chaos expansion," Water Air Soil Pollut., vol. 223, no. 7, pp. 4183-4197, 2012.

[35] Q. Shao, A. Younes, M. Fahs, and T. A. Mara, "Bayesian sparse polynomial chaos expansion for global sensitivity analysis," Comput. Methods Appl. Mech. Eng., vol. 318, pp. 474-496, 2017.
[36] R. L. Kashyap, "Optimal choice of AR and MA parts in autoregressive moving average models," IEEE Trans. Pattern Anal. Mach. Intell., vol. 4, no. 2, pp. 99-104, 1982.

[37] P. A. Guidault and T. Belytschko, "On the $L^{2}$ and the $H^{1}$ couplings for an overlapping domain decomposition method using Lagrange multipliers," Int. J. Num. Methods Eng., vol. 70, no. 3, pp. 322-350, 2007.

[38] L. Chamoin, S. Prudhomme, H. Ben Dhia, and J. T. Oden, "Ghost forces and spurious effects in atomic-to-continuum coupling methods by the arlequin approach," Int. J. Num. Methods Eng., vol. 83, no. 8-9, pp. 1081-1113, 2010.

[39] Q. Huang, H. Hu, K. Yu, M. Potier-Ferry, S. Belouettar, and N. Damil, "Macroscopic simulation of membrane wrinkling for various loading cases," Int. J. Solids Struct., vol. 64-65, pp. 246-258, 2015.

\section{Appendix A: Convergence analysis of the sample sizes}

The algorithm that we adopt to "train" the metamodel is efficient and accurate, and can get precise results at low sample sizes [35]. However, a relative larger data basically yields a more accurate estimation. In this part, we take the 2D-1D sandwich model as an example to investigate the effect of the sample size $N_{d}$ on results. The samples are drawn from the uniform distributions shown in Table 2 case 1 . And the model response $E r r_{f}$ is considered as output data. A SPCE model is constructed to approximate the input/output relations.

According to Shao et al. [35], a relative training error $\delta^{2}$ could be relied on to evaluate the quality of the constructed SPCE model. The relative training errors for different sample sizes are listed in Table 8. We can note that choosing the sample size as $2^{6}$ leads to a quite large relative error $\delta^{2}=0.117$, while increasing the model evaluations, the relative training error decreases. When $N_{d}$ equals $2^{9}$, the relative training error has been reduced to $\delta^{2}=0.046<0.05$, which is an acceptable level as suggested in [35].

Table 8. The relative training errors for different sample sizes in the 2D-1D sandwich model.

\begin{tabular}{lcccc}
\hline$N_{d}$ & $2^{6}$ & $2^{7}$ & $2^{8}$ & $2^{9}$ \\
\hline$\delta^{2}$ & 0.117 & 0.144 & 0.065 & 0.046 \\
\hline
\end{tabular}
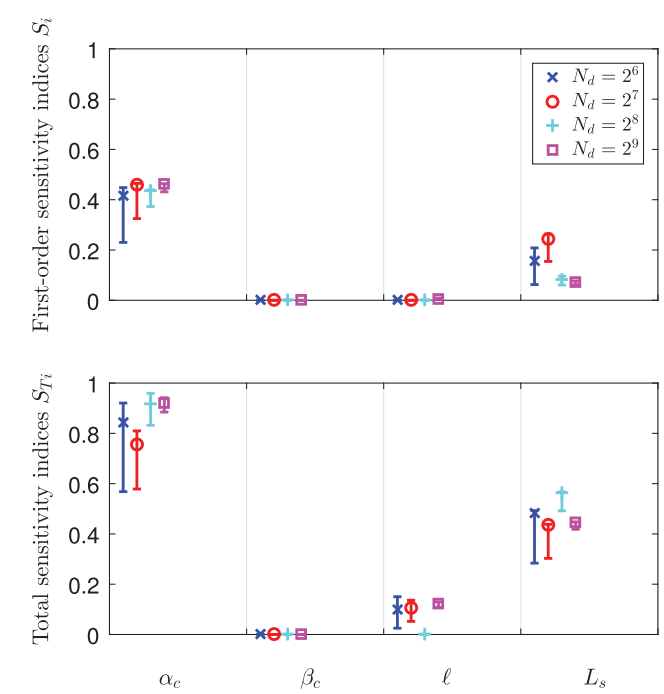

Figure $15.95 \%$ confidence intervals of the Sobol' indices with different sample sizes. 
Besides, the $95 \%$ credible intervals of both the first-order and total Sobol' indices estimates with different sample sizes are plotted in Figure 15. One clearly notes that with the sample size increases, the credible intervals become narrow and the maximum a posteriori estimates of the Sobol' indices converge. So choosing the sample size as $2^{9}$ for this case is reasonable.

\section{Appendix B: Formulations for the 2D-1D sandwich model}

The 2D FE model is discretized by 2D-Q8 elements, thus the displacement fields are described by

$$
\left\{\begin{array}{l}
u_{2 D}(x, z)=\left\langle\mathbf{N}_{\mathbf{u}}\right\rangle\left\{\mathbf{q}_{\mathbf{e}}\right\}=\left\langle\begin{array}{llllllllll}
N_{1} & 0 & \ldots & N_{8} & 0 & \rangle\left\langle u_{1}\right. & v_{1} & \ldots & u_{8} & v_{8}
\end{array}\right\rangle^{T}, \\
\left.v_{2 D}(x, z)=\left\langle\mathbf{N}_{\mathbf{v}}\right\rangle\left\{\mathbf{q}_{\mathbf{e}}\right\}=\begin{array}{lllllllll}
0 & N_{1} & \ldots & 0 & \left.N_{8}\right\rangle\left\langle u_{1}\right. & v_{1} & \ldots & u_{8} & v_{8}
\end{array}\right\rangle^{T},
\end{array}\right.
$$

where $u_{i}$ and $v_{i}(i=1, \ldots, 8)$ are respectively the longitudinal and the transversal nodal displacements of the $2 \mathrm{D}$ element. The shape functions $N_{i}$ can be found in [25], they are not presented here for brevity.

The kinematic field of the 1D sandwich beam is defined as follows:

$$
\begin{cases}u^{t}(x, z)=-\left(z-\frac{H_{1}}{2}\right) w_{, x}+\frac{H_{1}}{2} \beta(x) & \frac{H_{1}}{2}<z \leqslant \frac{H_{3}}{2}, \\ u^{m}(x, z)=z \beta(x) & \frac{-H_{1}}{2} \leqslant z \leqslant \frac{H_{1}}{2}, \\ u^{b}(x, z)=-\left(z+\frac{H_{1}}{2}\right) w_{, x}-\frac{H_{1}}{2} \beta(x) & -\frac{H_{3}}{2} \leqslant z<-\frac{H_{1}}{2}, \\ w(x, z)=w(x), & \\ \beta(x, z)=\beta(x), & \end{cases}
$$

where the superscripts $t, m$ and $b$ denote respectively the top, the middle and the bottom layer. Subscript $x$ preceded by comma stands for differentiation. The longitudinal displacement, the transverse displacement and the additional rotation of the normal to the mid-plane are respectively expressed by $u, w$ and $\beta$. The $1 \mathrm{D}$ sandwich beam is discretized by the two-node $1 \mathrm{D}$ element, in which $\beta$ is discretized by the Lagrange linear shape functions $\mathbf{N}_{\boldsymbol{\beta}}$ and $w$ by the Hermite shape functions $\mathbf{N}_{\mathbf{w}}$. Thus, the elemental unknowns are $\left\langle\phi_{\mathbf{e}}\right\rangle=\left\langle w_{1} \frac{\partial w_{1}}{\partial x} \beta_{1} w_{2} \frac{\partial w_{2}}{\partial x} \beta_{2}\right\rangle$.

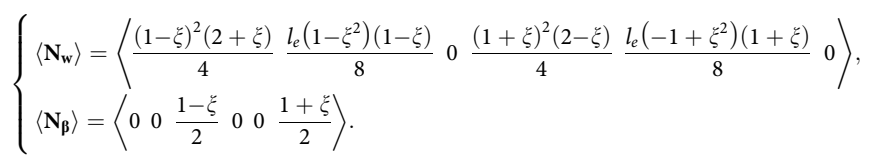

The field of Lagrange multipliers is the same as the displacement field of $1 \mathrm{D}$ sandwich model:

$$
\left\{\begin{array}{l}
\lambda_{u}^{t}=\left(-\left(z-\frac{H_{1}}{2}\right)\left\langle\mathbf{N}_{\mathbf{w}, \mathbf{x}}\right\rangle+\frac{H_{1}}{2}\left\langle\mathbf{N}_{\mathbf{\beta}}\right\rangle\right)\left\{\boldsymbol{\lambda}_{\mathbf{e}}\right\}, \\
\lambda_{u}^{m}=z\left\langle\mathbf{N}_{\boldsymbol{\beta}}\right\rangle\left\{\boldsymbol{\lambda}_{\mathbf{e}}\right\}, \\
\lambda_{u}^{b}=\left(-\left(z+\frac{H_{1}}{2}\right)\left\langle\mathbf{N}_{\mathbf{w}, \mathbf{x}}\right\rangle-\frac{H_{1}}{2}\left\langle\mathbf{N}_{\boldsymbol{\beta}}\right\rangle\right)\left\{\boldsymbol{\lambda}_{\mathbf{e}}\right\}, \\
\lambda_{w}=\left\langle\mathbf{N}_{\mathbf{w}}\right\rangle\left\{\boldsymbol{\lambda}_{\mathbf{e}}\right\},
\end{array}\right.
$$

where $\lambda_{u}$ and $\lambda_{w}$ are the longitudinal and transversal Lagrange multipliers, and $\lambda_{\mathrm{e}}$ is the element unknowns.

As only the longitudinal normal strain and stress under consideration, $\mathcal{K}_{1 D}, \mathcal{K}_{2 D}, \mathcal{F}_{1 D}$ and $\mathcal{F}_{2 D}$ which denote the element stiffness matrices and external force vectors of $1 \mathrm{D}$ and $2 \mathrm{D}$ sandwich element individually can be calculated easily by introducing the energy partition functions $\alpha_{c}$ and $\beta_{c}$. Besides, by introducing the coupling operator $H_{p}^{1}$ proposed by $\mathrm{Hu}$ et al. [25], the element coupling matrices can be calculated as:

1) $2 \mathrm{D}$ element

$$
\left\{\begin{array}{l}
\mathcal{C}_{2 D}^{t}=E_{2} \int_{-1}^{1} \int_{-1}^{1}\left(\left(-\left(z-\frac{H_{1}}{2}\right)\left\{\mathbf{N}_{\mathbf{w}, \mathbf{x}}\right\}+\frac{H_{1}}{2}\left\{\mathbf{N}_{\boldsymbol{\beta}}\right\}\right)\left\langle\mathbf{N}_{\mathbf{u}}\right\rangle+\left\{\mathbf{N}_{\mathbf{w}}\right\}\left\langle\mathbf{N}_{\mathbf{v}}\right\rangle+\mathcal{L}^{2}\left(-\left(z-\frac{H_{1}}{2}\right)\left\{\mathbf{N}_{\mathbf{w}, \mathbf{x} \mathbf{x}}\right\}+\frac{H_{1}}{2}\left\{\mathbf{N}_{\mathbf{\beta}, \mathbf{x}}\right\}\right)\left\langle\mathbf{N}_{\mathbf{u}, \mathbf{x}}\right\rangle\right) \mathcal{J} d \xi d \eta \\
\mathcal{C}_{2 D}^{m}=E_{1} \int_{-1}^{1} \int_{-1}^{1}\left(z\left\{\mathbf{N}_{\boldsymbol{\beta}}\right\}\left\langle\mathbf{N}_{\mathbf{u}}\right\rangle+\left\{\mathbf{N}_{\mathbf{w}}\right\}\left\langle\mathbf{N}_{\mathbf{v}}\right\rangle+\mathcal{L}^{2} z\left\{\mathbf{N}_{\mathbf{\beta}, \mathbf{x}}\right\}\left\langle\mathbf{N}_{\mathbf{u}, \mathbf{x}}\right\rangle\right) \mathcal{J} d \xi d \eta \\
\mathcal{C}_{2 D}^{b}=E_{2} \int_{-1}^{1} \int_{-1}^{1}\left(\left(-\left(z+\frac{H_{1}}{2}\right)\left\{\mathbf{N}_{\mathbf{w}, \mathbf{x}}\right\}-\frac{H_{1}}{2}\left\{N_{\beta}\right\}\right)\left\langle\mathbf{N}_{\mathbf{u}}\right\rangle+\left\{\mathbf{N}_{\mathbf{w}}\right\}\left\langle\mathbf{N}_{\mathbf{v}}\right\rangle+\mathcal{L}^{2}\left(-\left(z+\frac{H_{1}}{2}\right)\left\{\mathbf{N}_{\mathbf{w}, \mathbf{x} \mathbf{x}}\right\}-\frac{H_{1}}{2}\left\{\mathbf{N}_{\boldsymbol{\beta}, \mathbf{x}}\right\}\right)\left\langle\mathbf{N}_{\mathbf{u}, \mathbf{x}}\right\rangle\right) \mathcal{J} d \xi d \eta
\end{array}\right.
$$

2) $1 \mathrm{D}$ element

$$
\mathcal{C}_{1 D}=\mathcal{C}_{1 D}^{t}+\mathcal{C}_{1 D}^{m}+\mathcal{C}_{1 D}^{b}
$$

with

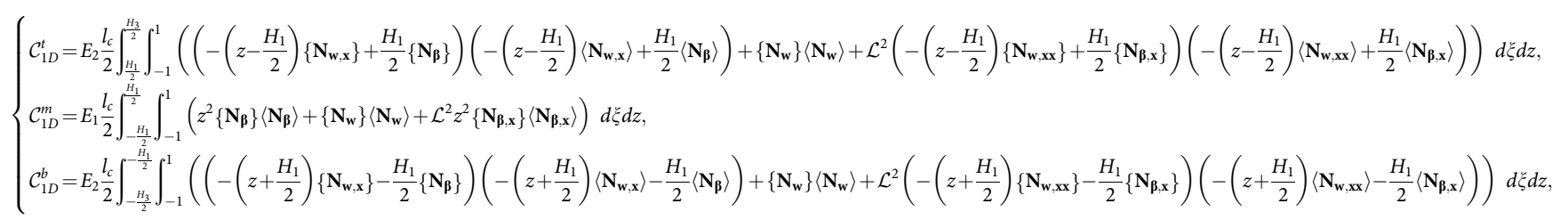

where $\mathcal{J}$ denotes the Jacobian determinant, the characteristic length $\mathcal{L} \in[0, L]$. And the summands in each integral are the longitudinal displacement, the transversal displacement and the longitudinal deformation couplings respectively.

After assembling all these elementary matrices, we can calculate the displacement field of the 2D-1D sandwich model that have the similar formulation as Eq. (5). 